\title{
Checklist of marine elasmobranchs of Colombia
}

\author{
Paola A. Mejía-Falla ${ }^{1,2, *}$, Andrés Felipe Navia ${ }^{1}$
}

Edited by

Juan Carlos Salcedo-Reyes

(salcedo.juan@javeriana.edu.co)

1. Fundación colombiana para la investigación y conservación de tiburones y rayas, SQUALUS.

Calle 10A No. 72-35,

Cali, Colombia.

2 Wildlife Conservation Society. Avenida 5 Norte No. 22N-11,

Cali, Colombia.

*pmejia@squalus.org

Received: 28-06-2018

Accepted: 24-01-2018

Published on line: 01-03-2019

Citation: Mejía-Falla PA, Navia AF. Checklist of marine elasmobranchs of Colombia, Universitas Scientiarum, 24 (1): 241-276, 2019.

doi: 10.11144/Javeriana.SC24-1.come

Funding:

N.A.

Electronic supplementary material: N.A.

OPEN ACCESS

\begin{abstract}
A review and update to the shark and ray species recorded for the marine waters of Colombia was carried out. A total of 206 species had been recorded in the literature, of which 138 species (76 sharks and 62 rays) could be confirmed from museum records, catches, photographs, and videos. No evidence was found for 25 species, but their distribution included neighboring countries, and they were therefore classified as possible based on distribution. Thirtysix species were classified as improbable based on distribution and seven more were considered as misidentifications. The inventory of confirmed species includes 57 genera (30 shark and 27 batoid genera) and 34 families (18 shark and 16 batoid families). There was notably an addition of 26 confirmed species since 2007 as well as numerous modifications to group systematics and taxonomy, especially for batoids. The total number of confirmed elasmobranchs represented $12.1 \%$ of species known worldwide, and could even reach $14.5 \%$, indicating that Colombia has one of the richest cartilaginous fish faunas in Latin America, behind Mexico and Brazil. This demonstrates that although Colombia cannot be considered an elasmobranch biodiversity hotspot or site of elasmobranch endemism globally, it does have good representativity of these species' biodiversity at the regional level, especially regarding amphi-American species.
\end{abstract}

Keywords: Biodiversity; sharks and batoids; species list; richness.

\section{Introduction}

Recent studies on shark and ray zoogeography in Colombia considered that the number of recorded species was high compared with the extension of the Colombian coasts, representing almost $15 \%$ of worldwide richness (Navia et al., 2016). Despite this high representativity, since the first mention 
of sharks in fish checklists of Colombia (Posada, 1909), few detailed studies have been carried out on the richness and distribution of these species in Colombia (García, 2017).

Numerous studies reporting first records of species or expansions of geographic range have been published in recent decades (e.g. Acero P \& Franke, 1995; Caldas et al., 2004; Acero et al., 2007; Grijalba-Bendeck \& Acevedo, 2009; Mejía-Falla \& Navia, 2009; Anguila et al., 2016 a,b; Acero et al., 2018), however, detailed listings of this group are rather scarce. The first specific checklists of elasmobranchs in Colombia were carried out by Mercado (1990), who included 61 species, and Mantilla (1998), who included 127 species. The latter author also included freshwater stingrays. Mejía-Falla et al. (2007), based on these checklists, on bibliographic references, and on physical and visual evidence, found that of 176 recorded species, only 121 could be considered confirmed for marine and freshwater environments of Colombia. The last chondrichthyan identification guide of Colombia included a total of 124 marine and freshwater elasmobranch species (Mejía-Falla et al., 2011).

A subsequent listing (Álvarez-León et al., 2013) based only on bibliographic compilation, and therefore lacking the evidence supports of the occurrence of the species, reported a total of 204 species of sharks and batoids for Colombia. This value greatly overestimates the elasmobranch richness of the country and contributes to perpetuating errors in the Colombian chondrichthyan biodiversity by including species as the great white shark (Carcharodon carcharias), the common thresher shark (Alopias vulpinus), electric rays as Discopyge tschudii and Narcine brasiliensis, and even sawfishes such as Pristis microdon, among others.

Since the publications by Mejía-Falla et al. (2007, 2011), there have been significant additions, and taxonomic and systematic modifications to this group internationally (Naylor et al., 2012; Last et al., 2016a; Weigmann, 2016). In particular, there have been changes to the classification of batoids and of some amphi-American sharks into different families and genera, and even the "resurrection" of families and species (Castro, 2011; Carvalho et al., 2016 a,b; Last et al., 2016 b,c; White \& Naylor, 2016; White \& Last, 2016 a,b,c). There have also been increases to the richness (Acero-P et al., 2016) and to the distribution of sharks and rays in Colombia (Navia et al., 2016; García, 2017), leading to current listings being out of date in terms of their composition locally (Mejía-Falla et al., 2007, 2011) and globally (Last et al., 2016 a; Weigmann, 2016), even more so regarding their taxonomic classification and systematics. 
The objective of the present study was to contribute to the update and consolidation of the marine cartilaginous fish checklist of Colombia, based on supporting evidence that would allow to confirm, question, or reject the presence of each analyzed species. Along with the updated checklist, annotations to modify the original lists were included as well as comments for species that present taxonomic or systematics problems.

\section{Materials and methods}

Construction and homogenization of cartilaginous fish databases

Based on checklists by Mejía-Falla et al. $(2007,2011)$ a baseline list of marine elasmobranch species of Colombia was created, to which newly available records were added (e.g. Gámez-Barrera et al., 2012; Acero-P et al., 2016, 2018; Anguila et al., 2016 a,b; García, 2017). Considering that the sole mention of a species in a document did not guarantee the presence of that species in the study area, a confirmation process for the presence of each species recorded in the initial list was performed. For this verification, the following activities were carried out:

1) A search of records of species in national and international ichthyological collections. Web pages were consulted, with an emphasis on spatial location data where each species was collected in order to validate its presence within the marine limits established for Colombian Pacific and Caribbean waters. For this study, it was assumed that a correct identification process had been undertaken for each of the recorded specimens in the ichthyological collections. However, when a species presented a record for Colombia but its natural distribution does not correspond to or close to Colombian waters, that record was considered a potential identification error.

2) The following national collections were consulted: INV-PEC: Marine Natural History Museum of Colombia (INVEMAR, Santa Marta), ICN-MHN: Natural Science Institute, Natural History Museum (Bogotá), IAvH: Ichthyological Collection of the Alexander von Humboldt Institute (Tunja), CIUA: Fish collection of the Universidad de Antioquia (Medellín), CICH-CHbCH: Hydrobiological Collection of Chocó, Universidad Tecnológica del Chocó (Quibdó), PNNG: Reference Marine Biological Collection of the Biological Station "Henry von Prahl” of the Gorgona Natural National Park (Gorgona Island), UNSSA: Universidad Nacional de Colombia in San Andrés (San Andrés). All the collections consulted, except UNSSA, are included in the National Registry of Biological Collections. Although the reference 
collection of the Universidad del Valle (CIRUV) was taken into account for a previous checklist (Mejía-Falla et al. 2007), it could not be included in the present study by the curator's request, as a revision of all specimens was underway. However, this did not affect the results of the present study, as all species previously confirmed in that collection were also reported by other museums, or there were records of capture or sightings.

The International collections reviewed were: AMNH: American Museum of Natural History (Washington), CAS: California Academy of Sciences (San Francisco), GCRL: Gulf Center Research Laboratory (Ocean Springs), FLMNH: Florida Museum of Natural History (Gainesville), NRM: Swedish Museum of Natural History (Sweden), USNM: Smithsonian National Museum of Natural History (Washington), SIO: Scripps Institution of Oceanography (San Diego, California), MNHN: Muséum National D'Histoire Naturelle (Paris), MCZ: Museum of Comparative Zoology, Harvard University (Cambridge), TCWC: Texas A\&M University, Cooperative Wildlife Collection (Texas), CMNFI: Canadian Museum of Nature Fish Collection, FMNH: Field Museum of Natural History (Zoology) Fish Collection (Chicago), SAIAB: The South African Institute for Aquatic Biodiversity (South Africa), AfroBIS: Iziko South African Museum, Shark Collection (South Africa).

Identification catalogues that included geolocation information in the species records (Robertson \& Allen 2015, Robertson et al., 2015) were also consulted, as well as the Global Biodiversity Information Facility (GBIF) and the Information System on Marine Biodiversity of Colombia (SIBM).

A search of capture records and in situ observations of individuals for different locations in Colombian Pacific and Caribbean waters. For these records several different information sources were consulted:

a) Documents related to checklists, updates or new records of fish species for Colombian Pacific and Caribbean waters or for particular areas within them, based on captures and/or visual observations, and for which there were descriptions and photographs that could validate the identification of the mentioned species.

b) Documents related to biological, ecological, and genetic studies of fish species within Colombian Pacific and Caribbean waters that included the diagnostic characteristics of the studied species and would therefore validate the presence of the species in the area. 
c) Photographic catalog of the Squalus Foundation, created from different projects carried out in Colombian Pacific and Caribbean waters, which allowed to validate the identity of the species and to record locations of capture or observation.

Reports of catches and visual observations were obtained from institutions such as the Squalus Foundation (SF), Malpelo Foundation (MF), EAT-Fishing consulting (EAT), Universidad del Valle (UV), and Marine and Coastal Research Institute, INVEMAR (INV). Geolocated records that appeared in "Shorefishes of the Tropical Eastern Pacific: online information system" (Record TEP) and "Shorefishes of the Greater Caribbean: online information system" (Record SGC) were also included, as well as records in published documents that included photographs of the specimens and allowed to validate their identity. Based on evidence found (museums, catches, visual observations, or literature records), the species cited in the present study were grouped into three large categories: 1 . Confirmed species: species that appeared in A) national and/or international museum records, or B) records of fisheries catches (catch) or in situ observations (visual record). 2. Unconfirmed species, possible based on distribution: Species that had been mentioned in different scientific documents, for which there was no physical evidence in any collection or geolocated visual confirmation. However, the known species distribution included the marine waters of neighboring countries, and therefore its presence in Colombian Pacific or Caribbean waters could not be rejected. 3. Unconfirmed species, improbable based on distribution: Species that, despite being included in marine fish checklists of Colombia or in regional identification guides, had distribution areas that did not encompass the study area, or even belonged to distant areas (e.g. Atlantic Ocean, Indo-Pacific, Indian Ocean, Mediterranean), to antitropical areas, or waters colder than those of the Eastern Pacific and Western Atlantic, which meant that their presence in Colombian Pacific or Caribbean waters would be improbable. The appearance of these species could have resulted from incorrect identifications or from being inadequately included based on previous checklists that did not verify sources. For unconfirmed species, two types of evidence were considered: C: regional or worldwide identification guides, and $\mathrm{D}$ : national references.

Since the taxonomy and systematics of this group has changed significantly over the past five years, it was necessary to update several orders, families, genera, and species. To do this were used as reference documents by Naylor et al. (2012), Carvalho et al. (2016 a,b), Last et al. (2016 a,b,c); White \& Naylor (2016), White \& Last (2016 a,b,c), and Weigmann (2016). This process allowed to present an updated checklist in terms of species nomenclature, and to avoid the duplication of species that could correspond to synonymies. 


\section{Results}

A total of 138 marine elasmobranchs were confirmed in this study, 76 sharks and 62 rays (Table 1), adding 26 species to the checklist by Mejía-Falla et al. (2007). This number of confirmed species for Colombia represents $12.1 \%$ of the world's total, as well as $30.5 \%$ of genera, $58.6 \%$ of families, and $80.0 \%$ of orders (Fig. 1). A total of 90 species (57 sharks and 33 rays) were confirmed for the Colombian Caribbean and 67 species (36 sharks and 31 rays; Table 1) were confirmed for the Colombian Pacific. Consequently, the representation percentage of genera and families was greater for the Caribbean than for the Pacific, whereas there were 11 orders found in each region (Fig. 1).

Twenty-five species (13 sharks and 12 batoids) could not be confirmed but were considered possible according to available records and the known distribution of the species (Table 2), whereas 36 species (15 sharks and 21 batoids) were catalogued as improbable due to their known distribution (Table 3). Although seven species presented collection records in locations within Colombia's Economic Exclusive Zone, there was only one record for each, or they were far from the known distribution of those species; they were therefore assumed to be incorrect identifications (Table 4).
Species
Genus
Families
Orders

\section{9}

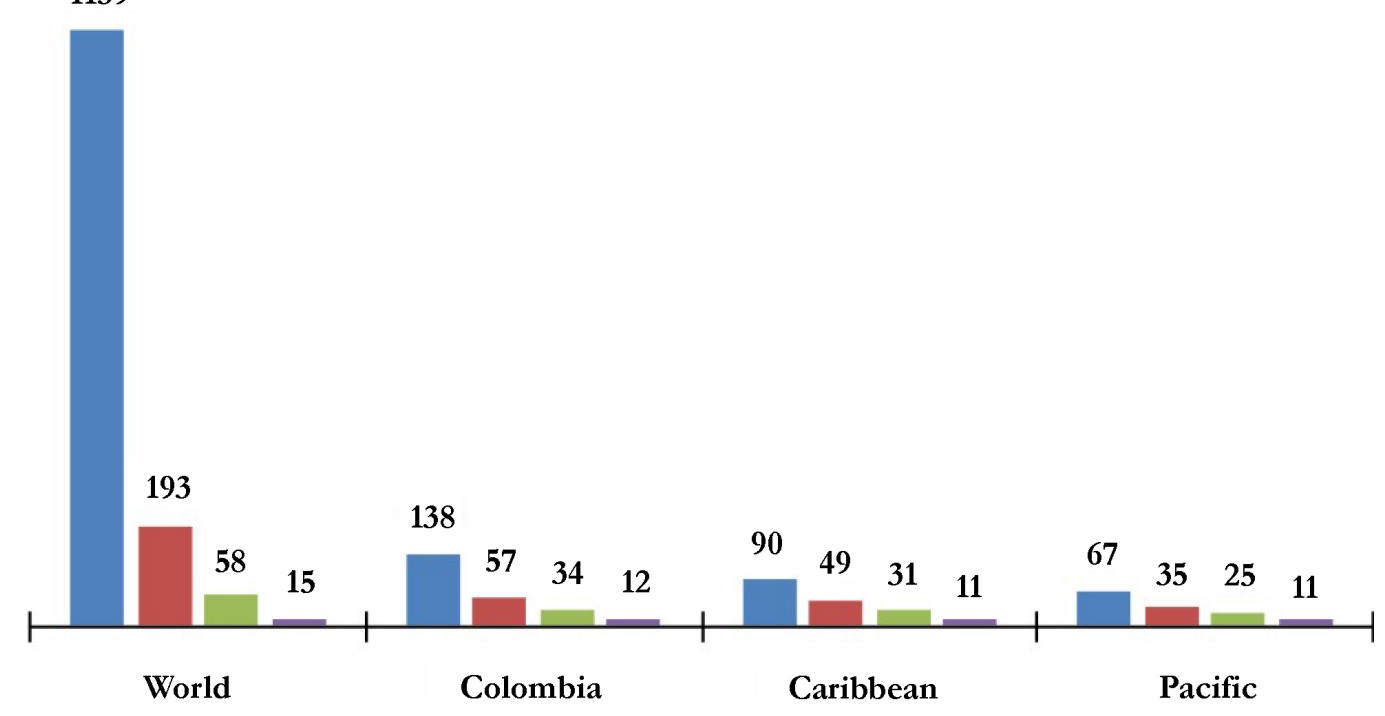

Figure 1. Numerical representation of the richness of orders, families, genera and species of elasmobranchs from Colombian Caribbean and Pacific, compared to the worldwide richness according to Weigmann (2016). 
Table 1. Species of confirmed elasmobranchs for the Colombian Pacific $(\mathrm{P})$ and Caribbean $(\mathrm{C})$, with their corresponding category (A: recorded in national and/or international museums; B: recorded in fisheries [catch] or in situ observations [visual record]) and type of support (see methods for details). Species added with respect to Mejía-Falla et al. $(2007,2011)$ are shaded in light blue, and species that presented modification in their systematics or taxonomy are shaded in light green. (*) specimens are presented in the indicated collection but have not yet been assigned a catalog number. (**) specimens correspond to holotypes.

No

Species

Category

Support

Area

Superorder Squalomorphii

\section{Order Heterodontiformes}

Family Heterodontidae

1 Heterodontus francisci

(Girard, 1855)

B Catch SF

$\mathrm{P}$

2 Heterodontus mexicanus

Taylor \& Castro-Aguirre, 1972

PNNG 1250; SIO 80-202,

A, B 80-203; AfrOBIS, Record TEP; P

Order Orectolobiformes

\section{Family Ginglymostomatidae}

3 Ginglymostoma cirratum

(Bonnaterre, 1788)

Catch SF

\begin{tabular}{|c|c|}
\hline 4 & $\begin{array}{l}\text { Ginglymostoma unami } \\
\text { del Moral-Flores et al., } 2015\end{array}$ \\
\hline
\end{tabular}

\section{Family Rhincodontidae}

\section{Rhincodon typus \\ Smith, 1828}

\section{Order Lamniformes}

\section{Family Alopiidae}
6 Alopias pelagicus
6 Nakamura, 1935
7 Alopias superciliosus
(Lowe, 1839)

\section{Family Lamnidae}

\author{
8 Isurus oxyrinchus \\ Isurus oxyrinchus
Rafinesque, 1810
}

$9 \quad \begin{aligned} & \text { Isurus paucus } \\ & \text { Guitart Manday, } 1966\end{aligned}$
A, B

IAvH-P 71; Record SGC;
PNNG 1061; INV-PEC 88117;

$\begin{array}{lll}\text { A, B } & \text { Record TEP; Record SGC; } & \text { P-C }\end{array}$

Visual record INV
B Record TEP;
Catch and visual record SF
Catch in Ospina (2001);
B Visual record in
Nieto et al. (2003); Catch SF

C

$\mathrm{P}$

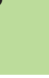




\section{Family Mitsukurinidae}

Jordan, 1898

Visual record in

B Grijalba-Bendeck \& Acevedo

(2009)

\section{Family Odontaspididae}

\section{Odontaspis ferox \\ (Risso, 1810)}

Catch in

B Anguila et al. (2016b); P-C

\section{Order Carcharhiniformes}

\section{Family Carcharhinidae}

12 Carcharbinus acronotus

(Poey, 1860)

13 Carcharbinus albimarginatus

(Rüppell, 1837)

14 Carcharbinus altimus

(Springer, 1950)

15 Carcharbinus brachyurus

(Günther, 1870)

16 Carcharbinus cerdale

Gilbert, 1898

17 Carcharbinus falciformis

(Müller \& Henle, 1839)

18 Carcharbinus galapagensis

(Snodgrass \& Heller, 1905)

19 Carcharhinus leucas

(Valenciennes, 1839)

20 Carcharbinus limbatus

(Valenciennes, 1839)

21 Carcharbinus longimanus

(Poey, 1861)

22 Carcharbinus perezii

(Poey, 1876)

23 Carcharhinus porosus

(Ranzani, 1839)

24 Galeocerdo cuvier

(Péron \& Lesueur, 1822)
A ICN-MHN 307

C

INV-PEC 92093, 88200;

A, B PNNG 1016, 1261;

Record TEP; Visual record SF

A, B SIO 62-205; Record TEP P-C

A USNM $232768 \quad$ P

FMNH 59346; CAS 56615;

A, B USNM 206825, 221211, 221214,

221227, 221211; Record TEP;

Catch SF

$\begin{array}{ll}\text { A, B } & \text { INV-PEC 7147, 8202; } \\ & \text { Record TEP; Visual record SF }\end{array}$

B Record TEP; Visual record SF P

USNM 206995; CMNFI 1974-

A, B 0095.1; Record TEP; Catch and P-C visual record $\mathrm{SF}$

INV-PEC 88108, 91102, 88130, 92048, 91099, 89037, 91109;

A, B PNNG 1264; Record TEP;

P-C

Record SGC; Catch and visual record SF

USNM 221232; CAS 66823,

\section{A, B SAIAB 39375; MCZ 165837; P-C}

Record TEP; Record SGC;

Visual record SF

A, B FLMNH 24416; Record SGC;

Visual record SF

C

INV-PEC 1732; CIUA 7, 3350;

Record SGC

C

INV-PEC 91008; PNNG 1257;

A, B Record TEP; Record SGC;

Catch and visual record SF 


\begin{tabular}{|c|c|c|c|c|}
\hline 25 & $\begin{array}{l}\text { Nasolamia velox } \\
\text { (Gilbert, 1898) }\end{array}$ & A, B & $\begin{array}{l}\text { USNM 206824; INV-PEC 93 } \\
\text { 90075, 87071, 90109; } \\
\text { PNNG 1266, 1269; } \\
\text { Record TEP }\end{array}$ & $\mathrm{P}$ \\
\hline 26 & $\begin{array}{l}\text { Negaprion brevirostris } \\
\text { (Poey, 1868) }\end{array}$ & B & $\begin{array}{l}\text { Record TEP; Record SGC; } \\
\text { Visual record SF }\end{array}$ & P-C \\
\hline 27 & $\begin{array}{l}\text { Prionace glauca } \\
\text { (Linnaeus, 1758) }\end{array}$ & $\mathrm{A}, \mathrm{B}$ & $\begin{array}{l}\text { ICN-MHN-477; } \\
\text { Catch and visual record SF }\end{array}$ & P-C \\
\hline 28 & $\begin{array}{l}\text { Rhizoprionodon lalandii } \\
\text { (Valenciennes, 1839) }\end{array}$ & A, B & $\begin{array}{l}\text { INV-PEC 5211; } \\
\text { FLMNH 224380, 28004, } \\
\text { USNM 221373; } \\
\text { Record SGC }\end{array}$ & C \\
\hline 29 & $\begin{array}{l}\text { Rhizoprionodon longurio } \\
\text { (Jordan \& Gilbert, 1882) }\end{array}$ & A, B & $\begin{array}{l}\text { INV-PEC 88005, 90071, } \\
\text { 90147, 90149, 91010, 91032; } \\
\text { PNNG 1265, 91032; } \\
\text { USNM 206823; Record TEP; } \\
\text { Catch SF }\end{array}$ & $\mathrm{P}$ \\
\hline 30 & $\begin{array}{l}\text { Rhizoprionodon porosus } \\
\text { (Poey, 1861) }\end{array}$ & A, B & $\begin{array}{l}\text { CIUA 9; FLMNH 28002, } \\
\text { USNM 221208, 221242; } \\
\text { Record SGC }\end{array}$ & C \\
\hline 31 & $\begin{array}{l}\text { Triaenodon obesus } \\
\text { (Rüppell, 1837) }\end{array}$ & A, B & $\begin{array}{l}\text { INV-PEC 87047, 87078; } \\
\text { PNNG 1018; Record TEP; } \\
\text { Visual record SF }\end{array}$ & $\mathrm{P}$ \\
\hline
\end{tabular}

\section{Family Scyliorhinidae}

\begin{tabular}{|c|c|c|c|c|}
\hline 32 & $\begin{array}{l}\text { Apristurus canutus } \\
\text { Springer \& Heemstra, } 1979\end{array}$ & A & USNM 221254 & $\mathrm{C}$ \\
\hline 33 & $\begin{array}{l}\text { Apristurus parvipinnis } \\
\text { Springer \& Heemstra, } 1979\end{array}$ & A & $\begin{array}{l}\text { USNM 201906, 221499, 268106; } \\
\text { TCWC } 33351.18\end{array}$ & $\mathrm{C}$ \\
\hline 34 & $\begin{array}{l}\text { Apristurus riveri } \\
\text { Bigelow \& Schroeder, } 1944\end{array}$ & A & USNM 221534 & $\mathrm{C}$ \\
\hline 35 & $\begin{array}{l}\text { Cephalurus cephalus* } \\
\text { (Gilbert, 1892) }\end{array}$ & A & INVPEC & $\mathrm{C}$ \\
\hline 36 & $\begin{array}{l}\text { Galeus arae } \\
\text { (Nichols, 1927) }\end{array}$ & A & USNM 402345 & $\mathrm{C}$ \\
\hline 37 & $\begin{array}{l}\text { Galeus cadenati } \\
\text { Springer, } 1966\end{array}$ & A & USNM 221368 & $\mathrm{C}$ \\
\hline 38 & $\begin{array}{l}\text { Schroederichthys maculatus } \\
\text { Springer, } 1966\end{array}$ & A & USNM 221650 & $\mathrm{C}$ \\
\hline 39 & $\begin{array}{l}\text { Scyliorhinus boa } \\
\text { Goode \& Bean, } 1896\end{array}$ & A & $\begin{array}{l}\text { INV-PEC 4556, 2430; } \\
\text { FLMNH 222965, 224213, 27979; } \\
\text { USNM 221563, } 221532\end{array}$ & $\mathrm{C}$ \\
\hline 40 & $\begin{array}{l}\text { Scyliorbinus baeckelii } \\
\text { (Miranda-Ribeiro, 1907) }\end{array}$ & B & Record SGC & $\mathrm{C}$ \\
\hline 41 & $\begin{array}{l}\text { Scyliorbinus hesperius } \\
\text { Springer, } 1966\end{array}$ & A & $\begin{array}{l}\text { FLMNH 27980, 223244; } \\
\text { USNM 221654, } 402344\end{array}$ & $\mathrm{C}$ \\
\hline 42 & $\begin{array}{l}\text { Scyliorbinus retifer } \\
\text { (Garman, 1881) }\end{array}$ & B & Record SGC & $\mathrm{C}$ \\
\hline
\end{tabular}




\begin{tabular}{|c|c|c|c|c|}
\hline 43 & $\begin{array}{l}\text { Scyliorbinus torrei } \\
\text { Howell Rivero, } 1936\end{array}$ & A & USNM 221560 & $\mathrm{C}$ \\
\hline & \multicolumn{4}{|l|}{ Family Sphyrnidae } \\
\hline 44 & $\begin{array}{l}\text { Sphyrna corona } \\
\text { Springer, } 1940\end{array}$ & A, B & $\begin{array}{l}\text { USNM 206819, 206987, } \\
\text { 222044, 222055; Record TEP; } \\
\text { Catch SF }\end{array}$ & $\mathrm{P}$ \\
\hline 45 & $\begin{array}{l}\text { Sphyrna lewini } \\
\text { (Griffith \& Smith, 1834) }\end{array}$ & A, B & $\begin{array}{l}\text { CICH-CHbCH 217; } \\
\text { NRM 13052, Record TEP; } \\
\text { Catch and visual record SF }\end{array}$ & $\mathrm{P}-\mathrm{C}$ \\
\hline 46 & $\begin{array}{l}\text { Sphyrna media } \\
\text { Springer, } 1940\end{array}$ & B & $\begin{array}{l}\text { Record TEP; } \\
\text { Catch and visual record SF; }\end{array}$ & $\mathrm{P}-\mathrm{C}$ \\
\hline 47 & $\begin{array}{l}\text { Sphyrna mokarran } \\
\text { (Rüppell, 1837) }\end{array}$ & B & Record TEP; Visual record SF & P-C \\
\hline 48 & $\begin{array}{l}\text { Sphyrna tiburo } \\
\text { (Linnaeus, 1758) }\end{array}$ & A, B & $\begin{array}{l}\text { INV-PEC 3668, 6151; IAvH-P 64; } \\
\text { USNM 222050, 222051, } 222035 \text {, } \\
\text { 94754; Catch and visual record SF }\end{array}$ & $\mathrm{P}-\mathrm{C}$ \\
\hline 49 & $\begin{array}{l}\text { Sphyrna tudes } \\
\text { (Valenciennes, 1822) }\end{array}$ & A, B & INV-PEC 7058; Record SGC & $\mathrm{C}$ \\
\hline \multirow[t]{2}{*}{50} & $\begin{array}{l}\text { Sphyrna yygaena } \\
\text { (Linnaeus, } 1758 \text { ) }\end{array}$ & B & Record TEP & $\mathrm{P}$ \\
\hline & \multicolumn{4}{|l|}{ Family Triakidae } \\
\hline 51 & $\begin{array}{l}\text { Mustelus canis } \\
\text { (Mitchill, 1815) }\end{array}$ & A & UNSSA* & $\mathrm{C}$ \\
\hline 52 & $\begin{array}{l}\text { Mustelus dorsalis } \\
\text { Gill, } 1864\end{array}$ & B & Record TEP & $\mathrm{P}$ \\
\hline 53 & $\begin{array}{l}\text { Mustelus benlei } \\
\text { (Gill, 1863) }\end{array}$ & $A, B$ & $\begin{array}{l}\text { INV-PEC 90121, 90129, 91067, } \\
\text { 90113, 92200, 91070, 90138, } \\
\text { 92040, 91128, 91078, 91071, } \\
\text { 91022, 92042; PNNG 681, 682, } \\
\text { 704, 713, 988, 1005, 1034, 1040, } \\
\text { 1071, 1080, 1249, 1253, 1383; } \\
\text { Record TEP; Catch SF }\end{array}$ & $\mathrm{P}$ \\
\hline 54 & $\begin{array}{l}\text { Mustelus bigmani } \\
\text { Springer \& Lowe, } 1963\end{array}$ & A & FLMNH 28000 & $\mathrm{C}$ \\
\hline 55 & $\begin{array}{l}\text { Mustelus lunulatus } \\
\text { Jordan \& Gilbert, } 1882\end{array}$ & A, B & $\begin{array}{l}\text { INV-PEC 92029, 90123, 91096, } \\
\text { 92047; PNNG 963, 1259, 1270, } \\
\text { 1275; USNM 206820, 206821, } \\
\text { 206822; Record TEP; Catch SF }\end{array}$ & $\mathrm{P}$ \\
\hline 56 & $\begin{array}{l}\text { Mustelus minicanis } \\
\text { Heemstra, } 1997\end{array}$ & A & USNM 207962, 208016 & $\mathrm{C}$ \\
\hline \multirow[t]{3}{*}{57} & $\begin{array}{l}\text { Mustelus norrisi } \\
\text { Springer, } 1939\end{array}$ & A & FLMNH 101345; USNM 201920 & $\mathrm{C}$ \\
\hline & Order Hexanchiformes & & & \\
\hline & Family Hexanchidae & & & \\
\hline 58 & $\begin{array}{l}\text { Heptranchias perlo } \\
\text { (Bonnaterre, 1788) }\end{array}$ & A, B & $\begin{array}{l}\text { UNSSA*, }^{*} \\
\text { Catch in Anguila et al. (2016a) }\end{array}$ & $\mathrm{C}$ \\
\hline
\end{tabular}




\begin{tabular}{|c|c|c|c|c|}
\hline 59 & $\begin{array}{l}\text { Hexanchus griseus } \\
\text { (Bonnaterre, 1788) }\end{array}$ & B & Catch in Anguila et al. (2016b) & P-C \\
\hline 60 & $\begin{array}{l}\text { Hexanchus nakamurai } \\
\text { Teng, } 1962\end{array}$ & A & $\mathrm{UNSSA}^{*}$ & $\mathrm{C}$ \\
\hline 61 & $\begin{array}{l}\text { Notorynchus cepedianus } \\
\text { (Péron, 1807) }\end{array}$ & $A, B$ & $\begin{array}{l}\text { INV-PEC 90151, 88075; } \\
\text { PNNG 1262, 1263; } \\
\text { AfrOBIS; Record TEP }\end{array}$ & $\mathrm{P}$ \\
\hline
\end{tabular}

\section{Family Echinorhinidae}

$62 \begin{aligned} & \text { Echinorbinus brucus } \\ & \text { (Bonnaterre, 1788) }\end{aligned} \quad$ B $\quad$ Catch Anguila et al. (2016b) C

\section{Order Squaliformes}

\section{Family Centrophoridae}

63 Centrophorus granulosus

63 (Bloch \& Schneider, 1801)

A INV-PEC 2281; USNM $205781 \quad$ C

\section{Family Etmopteridae}

\section{Centroscyllium nigrum}

Garman, 1899

\section{Etmopterus bullisi}

Bigelow \& Schroeder, 1957

\section{Etmopterus carteri}

66 Springer \& Burgess, 1985

67 Etmopterus gracilispinis

67 Krefft, 1968

68 Etmopterus hillianus (Poey, 1861)

\section{Etmopterus perryi \\ 69 Springer \& Burgess, 1985}

70 Etmopterus robinsi

$70 \quad$ Schofield \& Burgess, 1997

\section{Etmopterus schultri}

71 Bigelow, Schroeder \& Springer, 1953

\section{Etmopterus virens}

72 Bigelow, Schroeder \& Springer, 1953

\section{Family Somniosidae}

73 Somniosus sp. Lesueur, 1818

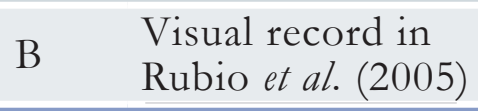

$\mathrm{P}$

AfrOBIS-SAMS-

SHARKS-003686

C

A $\begin{aligned} & \text { USNM 206090** 206091, 206092; C C } \\ & \text { FLMNH 40691 }\end{aligned}$

A FLMNH 27977

C

A TCWC 7360.06

C

INV-PEC 2435-37,

A USNM 206093**, 206094, 206095, C
202620, 206221;

FLMNH 27973, 40692, 40693

A FLMNH 27960

C

INV-PEC 2438-43, 8266, 8267;

FLMNH 27975, 27978;

A USNM 220394, 220425, 220443, C 268110, 268113;

TCWC 6511.02, 3789.14

FLMNH 27974, 27976;

A USNM 220450, 220448, 220454, C 220474, 220484

C




\section{Family Squalidae}

$74 \begin{aligned} & \text { Squalus cubensis } \\ & \text { Howell Rivero, } 1936\end{aligned}$

ANV-PEC 717, 2356;
USNM 220584, 220603

C

\section{Order Squatiniformes}

\section{Family Squatinidae}

\section{Squatina armata}

(Philippi, 1887)

A, B PNNG 1256; Catch SF

$\mathrm{P}$

\section{Squatina david}

76 Acero P., Tavera,

Anguila \& Hernández, 2016

INV-PEC 8250, 8252, 2186,

3988,4117

\section{Superorder Batoidei}

\section{Order Torpediniformes}

\section{Family Narcinidae}

1 Diplobatis colombiensis

Fechhelm \& McEachran, 1984

2 Diplobatis guamachensis

Martin-Salazar, 1957

3 Diplobatis ommata

(Jordan \& Gilbert, 1890)

$4 \quad$ Diplobatis picta

Palmer, 1950

\section{$5 \quad$ Narcine bancroftii \\ (Griffith \& Smith, 1834)}

6 Narcine entemedor

Jordan \& Starks, 1895

7 Narcine leoparda

7 Carvalho, 2001

8 Narcine vermiculata

\section{Family Torpedinidae}

9 Tetronarce nobiliana

(Bonaparte, 1835)
A USNM $232494^{* *}, 232929,226773$ C

INV-PEC 1934, 4435, 4434, 3928, 3929, 4106,

A 6233, 6234, 6235;

FLMNH 29870, 29871, 29872, 29876, 29880, 29888, 29889, 29890, 29891, 110043

B Record TEP P

USNM 222449; FLMNH 29869,

29871, 29878, 29877, 29879,

29891, 176815, 222289, 222337,

C

226509

INV-PEC 2124, 2224, 5201-

A, B 03; ICN-MHN 1372; FLMNH

228854; USNM 00222463 ,

C 00222496; Visual record SF

INV-PEC 6778; CAS 58354;

USNM 206986, 206997,

222466; Record TEP;

P

Catch and visual record SF

INV-PEC 6731; USNM 222198,

$\begin{array}{ll}\text { A, B } & \text { 222199, 222200, 222498, } \\ & \text { 222500, 235919; Record TEP; }\end{array}$

Catch and visual record SF

A GCRL 5145

P 


\begin{tabular}{|c|c|c|c|c|}
\hline 10 & $\begin{array}{l}\text { Tetronarce tremens } \\
\text { (de Buen, 1959) }\end{array}$ & A & INV-PEC sin número & $\mathrm{P}$ \\
\hline 11 & $\begin{array}{l}\text { Torpedo andersoni } \\
\text { Bullis, } 1962\end{array}$ & $\mathrm{~B}$ & Visual record SF & $\mathrm{C}$ \\
\hline & \multicolumn{4}{|l|}{ Order Rhinopristiformes } \\
\hline & \multicolumn{4}{|l|}{ Family Pristidae } \\
\hline 12 & $\begin{array}{l}\text { Pristis pristis } \\
\text { (Linnaeus, 1758) }\end{array}$ & $\mathrm{B}$ & $\begin{array}{l}\text { Saw records in } \\
\text { Gómez-Rodríguez et al. (2014); } \\
\text { Record TEP; Catch and visual } \\
\text { record SF }\end{array}$ & $\mathrm{P}-\mathrm{C}$ \\
\hline 13 & $\begin{array}{l}\text { Pristis pectinata } \\
\text { Latham, } 1794\end{array}$ & $\mathrm{~B}$ & $\begin{array}{l}\text { Saw records in } \\
\text { Gómez-Rodríguez et al. (2014) }\end{array}$ & $\mathrm{C}$ \\
\hline & \multicolumn{4}{|l|}{ Family Rhinobatidae } \\
\hline 14 & $\begin{array}{l}\text { Pseudobatos glaucostigma } \\
\text { (Jordan \& Gilbert, 1883) }\end{array}$ & $\mathrm{B}$ & Record TEP & $\mathrm{P}$ \\
\hline 15 & $\begin{array}{l}\text { Pseudobatos leucorbynchus } \\
\text { (Günther, 1866) }\end{array}$ & $\mathrm{A}, \mathrm{B}$ & $\begin{array}{l}\text { INV-PEC 4972; } \\
\text { USNM 206816, 206817, 206818; } \\
\text { Record TEP; } \\
\text { Catch and visual record SF }\end{array}$ & $\mathrm{P}$ \\
\hline 16 & $\begin{array}{l}\text { Pseudobatos percellens } \\
\text { (Walbaum, 1792) }\end{array}$ & A & $\begin{array}{l}\text { ICN-MHN 3769; INV-PEC 3963; } \\
\text { IAvH-P 131; FLMNH 37005; } \\
\text { USNM 222085, } 222078\end{array}$ & C \\
\hline 17 & $\begin{array}{l}\text { Pseudobatos planiceps } \\
\text { (Garman, 1880) }\end{array}$ & $\mathrm{A}, \mathrm{B}$ & TCWC 12113.02; Record TEP & $\mathrm{P}$ \\
\hline 18 & $\begin{array}{l}\text { Pseudobatos prabli } \\
\text { (Acero P. \& Franke, 1995) }\end{array}$ & $\mathrm{A}, \mathrm{B}$ & $\begin{array}{l}\text { ICN-MHN 4049; Record TEP; } \\
\text { Catch and visual record SF }\end{array}$ & $\mathrm{P}$ \\
\hline & \multicolumn{4}{|l|}{ Family Trygonorrhinidae } \\
\hline 19 & $\begin{array}{l}\text { Zapteryx xyster } \\
\text { Jordan \& Evermann, } 1896\end{array}$ & A, B & $\begin{array}{l}\text { PNNG 1258, 1260; } \\
\text { USNM 206992, 222091, 222100; } \\
\text { Record TEP; } \\
\text { Catch and visual record SF }\end{array}$ & $\mathrm{P}$ \\
\hline & \multicolumn{4}{|l|}{ Order Rajiformes } \\
\hline & \multicolumn{4}{|l|}{ Family Rajidae } \\
\hline 20 & $\begin{array}{l}\text { Breviraja nigriventralis } \\
\text { McEachran \& Matheson, } 1985\end{array}$ & A & $\begin{array}{l}\text { INV-PEC 2451-53, 8274; } \\
\text { FLMNH 29854; } \\
\text { MCZ 164855, 51809, 51019; } \\
\text { TCWC } 6511.26\end{array}$ & $\mathrm{C}$ \\
\hline 21 & $\begin{array}{l}\text { Breviraja spinosa } \\
\text { Bigelow \& Schroeder, } 1950\end{array}$ & A & TCWC 2807.01; MCZ 59234 & $\mathrm{C}$ \\
\hline 22 & $\begin{array}{l}\text { Dactylobatus clarkii } \\
\text { (Bigelow \& Schroeder, 1958) }\end{array}$ & A & $\begin{array}{l}\text { INV-PEC 2454-55, USNM } \\
222213 ; \text { MCZ 51069, 51810, } \\
48999\end{array}$ & $\mathrm{C}$ \\
\hline 23 & $\begin{array}{l}\text { Dipturus bullisi } \\
\text { (Bigelow \& Schroeder, 1962) }\end{array}$ & A & $\begin{array}{l}\text { INV-PEC 2456, 3277-78; } \\
\text { FLMNH 29860; MCZ 59236 }\end{array}$ & $\mathrm{C}$ \\
\hline
\end{tabular}




\begin{tabular}{|c|c|c|c|c|}
\hline 24 & $\begin{array}{l}\text { Dipturus garricki } \\
\text { (Bigelow \& Schroeder, 1958) }\end{array}$ & A & $\begin{array}{l}\text { INV-PEC } 2457, \\
\text { FLMNH } 123764\end{array}$ & C \\
\hline 25 & $\begin{array}{l}\text { Dipturus teevani } \\
\text { (Bigelow \& Schroeder, 1951) }\end{array}$ & A & FLMNH 29856 & C \\
\hline 26 & $\begin{array}{l}\text { Rostroraja cervigoni } \\
\text { (Bigelow \& Schroeder, 1964) }\end{array}$ & A & $\begin{array}{l}\text { INV-PEC 2184; } \\
\text { MCZ 51037, 51053 }\end{array}$ & $\mathrm{C}$ \\
\hline 27 & $\begin{array}{l}\text { Rostroraja equatorialis } \\
\text { (Jordan \& Bollman, 1890) }\end{array}$ & A & $\begin{array}{l}\text { INV-PEC 4971, USNM 222220, } \\
\text { 222215; TCWC 6531.01, } 15483.01\end{array}$ & $\mathrm{P}$ \\
\hline 28 & $\begin{array}{l}\text { Rostroraja velezi } \\
\text { (Chirichigno, 1973) }\end{array}$ & A, B & $\begin{array}{l}\text { INV-PEC 88033, 92094; } \\
\text { PNNG 1268; Record TEP; } \\
\text { Catch and visual record SF }\end{array}$ & $\mathrm{P}$ \\
\hline 29 & $\begin{array}{l}\text { Rajella fuliginea } \\
\text { (Bigelow \& Schroeder, 1954) }\end{array}$ & A & FLMNH 29858 & $\mathrm{C}$ \\
\hline \multirow[t]{2}{*}{30} & $\begin{array}{l}\text { Rajella nigerrima } \\
\text { (de Buen, 1960) }\end{array}$ & A & TCWC 3885.01 & $\mathrm{P}$ \\
\hline & \multicolumn{4}{|l|}{ Family Gurgesiellidae } \\
\hline 31 & $\begin{array}{l}\text { Fenestraja plutonia } \\
\text { (Garman, 1881) }\end{array}$ & A & TCWC 2572.01 & C \\
\hline 32 & $\begin{array}{l}\text { Fenestraja sinusmexicanus } \\
\text { (Bigelow \& Schroeder, 1950) }\end{array}$ & A, B & $\begin{array}{l}\text { TCWC 7360.23; } \\
\text { MCZ 51068; Record SGC }\end{array}$ & $\mathrm{C}$ \\
\hline 33 & $\begin{array}{l}\text { Gurgesiella atlantica } \\
\text { (Bigelow \& Schroeder, 1962) }\end{array}$ & A & $\begin{array}{l}\text { INV-PEC 1351, 2458-60, 8275, } \\
\text { 8276; FLMNH 29859, 222933, } \\
\text { 117467; USNM 222261, 222258; } \\
\text { TWCW 7360.22, 2735.01; } \\
\text { MCZ 51798, 51805, 164871 }\end{array}$ & $\mathrm{C}$ \\
\hline & \multicolumn{4}{|l|}{ Family Anacanthobatidae } \\
\hline 34 & $\begin{array}{l}\text { Schroederobatis americana } \\
\text { (Bigelow \& Schroeder, 1962) }\end{array}$ & A & $\begin{array}{l}\text { INV-PEC 1350, 2447, 2446, 2448, } \\
\text { 2449, 2450, 8273, 8268, 8269, } \\
\text { 8270, 8271, 8272, 7913; } \\
\text { FLMNH 29855, 29857, } 117468 ; \\
\text { USNM 222143, 222146; } \\
\text { MCZ 164854, 51815, 51807, } \\
\text { 49003, 48995, 48993; } \\
\text { TCWC } 6511.27,3353.05,5682.01\end{array}$ & $\mathrm{C}$ \\
\hline \multirow[t]{2}{*}{35} & $\begin{array}{l}\text { Springeria longirostris } \\
\text { (Bigelow \& Schroeder, 1962) }\end{array}$ & A & $\begin{array}{l}\text { TCWC } 7360.20,7374.04,7374.03 \text {, } \\
3789.18,3789.25\end{array}$ & $\mathrm{C}$ \\
\hline & \multicolumn{4}{|l|}{ Family Crurirajidae } \\
\hline 36 & $\begin{array}{l}\text { Cruriraja poeyi } \\
\text { Bigelow \& Schroeder, } 1948\end{array}$ & A & FLMNH 123765 & C \\
\hline \multirow[t]{3}{*}{37} & $\begin{array}{l}\text { Cruriraja rugosa } \\
\text { Bigelow \& Schroeder, } 1958\end{array}$ & A & $\begin{array}{l}\text { FLMNH 29851, 222752; } \\
\text { MCZ } 164867\end{array}$ & $\mathrm{C}$ \\
\hline & \multicolumn{4}{|l|}{ Order Myliobatiformes } \\
\hline & \multicolumn{4}{|l|}{ Family Dasyatidae } \\
\hline 38 & $\begin{array}{l}\text { Hypanus americanus } \\
\text { (Hildebrand \& Schroeder, 1928) }\end{array}$ & A & $\begin{array}{l}\text { PEC1483; ICN-MHN 4003; } \\
\text { FLMNH 10861, Visual record SF }\end{array}$ & C \\
\hline
\end{tabular}




\begin{tabular}{|c|c|c|c|c|}
\hline 39 & $\begin{array}{l}\text { Hypanus dipterurus } \\
\text { (Jordan \& Gilbert, 1880) }\end{array}$ & $\mathrm{A}, \mathrm{B}$ & $\begin{array}{l}\text { TCWC 13217.01; Record TEP; } \\
\text { Catch and visual record SF }\end{array}$ & $\mathrm{P}$ \\
\hline 40 & $\begin{array}{l}\text { Hypanus guttatus } \\
\text { (Bloch \& Schneider, 1801) }\end{array}$ & A & $\begin{array}{l}\text { INV-PEC 1733, 2334, 5443; } \\
\text { IAVH-P 138; NRM 24275, } 15806\end{array}$ & $\mathrm{C}$ \\
\hline 41 & $\begin{array}{l}\text { Hypanus longus } \\
\text { (Garman, 1880) }\end{array}$ & $\mathrm{A}, \mathrm{B}$ & $\begin{array}{l}\text { INV-PEC 91068, 91095, 91110; } \\
\text { PNNG 1252, 1271; } \\
\text { USNM 206990; Record TEP; } \\
\text { Catch and visual record SF }\end{array}$ & $\mathrm{P}$ \\
\hline 42 & $\begin{array}{l}\text { Pteroplatytrygon violacea } \\
\text { (Bonaparte, 1832) }\end{array}$ & B & Catch and visual record SF & $\mathrm{P}$ \\
\hline \multicolumn{5}{|c|}{ Family Gymnuridae } \\
\hline 43 & $\begin{array}{l}\text { Gymnura crebripunctata } \\
\text { (Peters, 1869) }\end{array}$ & A & USNM 222595, 206993 & $\mathrm{P}$ \\
\hline 44 & $\begin{array}{l}\text { Gymnura marmorata } \\
\text { (Cooper, 1864) }\end{array}$ & $\mathrm{B}$ & $\begin{array}{l}\text { Record TEP; Catch and visual } \\
\text { record SF }\end{array}$ & $\mathrm{P}$ \\
\hline \multicolumn{5}{|c|}{ Family Mobulidae } \\
\hline 45 & $\begin{array}{l}\text { Mobula birostris } \\
\text { (Walbaum, 1792) }\end{array}$ & $\mathrm{B}$ & Record TEP; Visual record SF & $\mathrm{P}-\mathrm{C}$ \\
\hline 46 & $\begin{array}{l}\text { Mobula munkiana } \\
\text { Notarbatolo-di Sciara, } 1987\end{array}$ & $\mathrm{~A}, \mathrm{~B}$ & $\begin{array}{l}\text { INV-PEC 89004; PNNG 1271; } \\
\text { Record TEP }\end{array}$ & $\mathrm{P}$ \\
\hline 47 & $\begin{array}{l}\text { Mobula thurstoni } \\
\text { (Lloyd, 1908) }\end{array}$ & $\mathrm{A}, \mathrm{B}$ & PNNG 1267; Record TEP & $\mathrm{P}$ \\
\hline \multicolumn{5}{|c|}{ Family Aetobatidae } \\
\hline 48 & $\begin{array}{l}\text { Aetobatus laticeps } \\
\text { (Gill, 1865) }\end{array}$ & $\mathrm{B}$ & $\begin{array}{l}\text { Record TEP; } \\
\text { Catch and visual record SF }\end{array}$ & $\mathrm{P}$ \\
\hline 49 & $\begin{array}{l}\text { Aetobatus narinari } \\
\text { (Euphrasen, } 1790 \text { ) }\end{array}$ & $\mathrm{A}, \mathrm{B}$ & $\begin{array}{l}\text { ICN-MHN 1339, 3779; } \\
\text { IAvH-P 132, 3067; Record SGC; } \\
\text { Visual record SF }\end{array}$ & $\mathrm{C}$ \\
\hline \multicolumn{5}{|c|}{ Family Rhinopteridae } \\
\hline 50 & $\begin{array}{l}\text { Rhinoptera bonasus } \\
\text { (Mitchill, 1815) }\end{array}$ & A & INV-PEC 1531; IAvH-P 125 & $\mathrm{C}$ \\
\hline 51 & $\begin{array}{l}\text { Rhinoptera brasiliensis } \\
\text { Müller, } 1836\end{array}$ & A & INV-PEC 615 & $\mathrm{C}$ \\
\hline 52 & $\begin{array}{l}\text { Rhinoptera steindachneri } \\
\text { Evermann \& Jenkins, } 1891\end{array}$ & $\mathrm{~A}, \mathrm{~B}$ & $\begin{array}{l}\text { PNNG 1274; Record TEP; } \\
\text { Catch and visual record SF }\end{array}$ & $\mathrm{P}$ \\
\hline \multicolumn{5}{|c|}{ Family Potamotrygonidae } \\
\hline 53 & $\begin{array}{l}\text { Styracura pacifica } \\
\text { (Beebe \& Tee-Van, 1941) }\end{array}$ & B & $\begin{array}{l}\text { Visual record in } \\
\text { Ross \& Schäfer }(2000)\end{array}$ & $\mathrm{P}$ \\
\hline 54 & $\begin{array}{l}\text { Styracura schmardae } \\
\text { (Werner, 1904) }\end{array}$ & $\mathrm{A}, \mathrm{B}$ & $\begin{array}{l}\text { INV-PEC } 1734, \text { IAvH-P } 135 ; \\
\text { Visual record SF }\end{array}$ & $\mathrm{C}$ \\
\hline \multicolumn{5}{|c|}{ Family Urotrygonidae } \\
\hline 55 & $\begin{array}{l}\text { Urobatis tumbesensis } \\
\text { (Chirichigno \& McEachran, 1979) }\end{array}$ & $\mathrm{B}$ & $\begin{array}{l}\text { Visual record in } \\
\text { Mejía-Falla \& Navia (2009) }\end{array}$ & $\mathrm{P}$ \\
\hline
\end{tabular}




\begin{tabular}{|c|c|c|c|c|}
\hline 56 & $\begin{array}{l}\text { Urobatis halleri } \\
\text { (Cooper, } 1863 \text { ) }\end{array}$ & B & Record TEP; Visual record SF & $\mathrm{P}$ \\
\hline 57 & $\begin{array}{l}\text { Urobatis jamaicensis } \\
\text { (Cuvier, 1816) }\end{array}$ & A & $\begin{array}{l}\text { INV-PEC 1482, 4037, 4124, 3792; } \\
\text { IAvH-P 101; GCRL15263 }\end{array}$ & $\mathrm{C}$ \\
\hline 58 & $\begin{array}{l}\text { Urotrygon aspidura } \\
\text { (Jordan \& Gilbert, 1882) }\end{array}$ & A, B & $\begin{array}{l}\text { USNM 222705; CIUA 4749, } 4752 \text {, } \\
\text { 4754; Record TEP; Catch SF }\end{array}$ & $\mathrm{P}$ \\
\hline 59 & $\begin{array}{l}\text { Urotrygon chilensis } \\
\text { (Günther, 1872) }\end{array}$ & A, B & $\begin{array}{l}\text { USNM 222628, 222638; } \\
\text { Record TEP }\end{array}$ & $\mathrm{P}$ \\
\hline 60 & $\begin{array}{l}\text { Urotrygon munda } \\
\text { Gill, } 1863\end{array}$ & $\mathrm{~A}, \mathrm{~B}$ & USNM 206994; Record TEP & $\mathrm{P}$ \\
\hline 61 & $\begin{array}{l}\text { Urotrygon rogersi } \\
\text { (Jordan \& Starks, 1895) }\end{array}$ & A, B & $\begin{array}{l}\text { INV-PEC 6715; CIUA 4748, } \\
\text { 4750; Record TEP; Catch SF }\end{array}$ & $\mathrm{P}$ \\
\hline 62 & $\begin{array}{l}\text { Urotrygon venezuelae } \\
\text { Schultz, } 1949\end{array}$ & A & $\begin{array}{l}\text { ICN-MHN 11099; AMNH 55623, } \\
\text { NRM 16282, GCRL 15264; } \\
\text { TCWC 7057.01, 7058.02 }\end{array}$ & $\mathrm{C}$ \\
\hline
\end{tabular}

Table 2. List of elasmobranch species not confirmed, but possible by distribution for the Colombian Pacific (P) or Caribbean (C). The type (C, D) and reference of support are indicated (see methods for details). Species with modifications in their systematics or taxonomy are shaded in light green.

\begin{tabular}{|c|c|c|c|c|}
\hline No & Category & $\begin{array}{c}\text { Support } \\
\text { type }\end{array}$ & Reference & Area \\
\hline & \multicolumn{4}{|l|}{ Superorder Squalomorphi } \\
\hline & \multicolumn{4}{|l|}{ Order Lamniformes } \\
\hline & \multicolumn{4}{|l|}{ Family Alopiidae } \\
\hline \multirow[t]{2}{*}{1} & $\begin{array}{l}\text { Alopias vulpinus } \\
\text { (Bonnaterre, 1788) }\end{array}$ & $\mathrm{D}$ & Álvarez-León et al., 2013 & $\mathrm{P}$ \\
\hline & \multicolumn{4}{|l|}{ Familia Cetorhinidae } \\
\hline \multirow[t]{2}{*}{2} & $\begin{array}{l}\text { Cetorbinus maximus } \\
\text { (Gunnerus, 1765) }\end{array}$ & $\mathrm{C}$ & Skomal et al., 2009 & $\mathrm{C}$ \\
\hline & \multicolumn{4}{|l|}{ Family Pseudocarchariidae } \\
\hline \multirow[t]{3}{*}{3} & $\begin{array}{l}\text { Pseudocarcharias kamoharai } \\
\text { (Matsubara, 1936) }\end{array}$ & $\mathrm{C}$ & Compagno et al., 1995 & $\mathrm{P}$ \\
\hline & \multicolumn{4}{|l|}{ Order Carcharhiniformes } \\
\hline & \multicolumn{4}{|l|}{ Family Carcharhinidae } \\
\hline 4 & $\begin{array}{l}\text { Carcharbinus brevipinna } \\
\text { (Valenciennes, 1839) }\end{array}$ & $\mathrm{C}$ & Cervigón \& Alcalá, 1999 & $\mathrm{C}$ \\
\hline
\end{tabular}




\begin{tabular}{|c|c|c|c|c|}
\hline 5 & $\begin{array}{l}\text { Carcharbinus obscurus } \\
\text { (Lesueur, 1818) }\end{array}$ & $\mathrm{D}$ & Martínez, 1978 & $\mathrm{C}$ \\
\hline 6 & $\begin{array}{l}\text { Carcharbinus plumbeus } \\
\text { (Nardo, 1827) }\end{array}$ & $\mathrm{D}$ & Dahl, 1964 & $\mathrm{C}$ \\
\hline \multicolumn{5}{|c|}{ Family Scyliorhinidae } \\
\hline 7 & $\begin{array}{l}\text { Apristurus nasutus } \\
\text { de Buen, } 1959\end{array}$ & $\mathrm{D}$ & Rubio, 1987 & $\mathrm{P}$ \\
\hline \multicolumn{5}{|c|}{ Family Triakidae } \\
\hline 8 & $\begin{array}{l}\text { Mustelus mento } \\
\text { Cope, } 1877\end{array}$ & $\mathrm{D}$ & Rubio, 1987 & $\mathrm{P}$ \\
\hline 9 & $\begin{array}{l}\text { Mustelus whitneyi } \\
\text { Chirichigno F, } 1973\end{array}$ & $\mathrm{D}$ & Rubio, 1987 & $\mathrm{P}$ \\
\hline 10 & $\begin{array}{l}\text { Triakis acutipinna } \\
\text { Kato, } 1968\end{array}$ & $\mathrm{D}$ & Rubio, 1987 & $\mathrm{P}$ \\
\hline \multicolumn{5}{|c|}{ Order Echinorhiniformes } \\
\hline \multicolumn{5}{|c|}{ Family Echinorhinidae } \\
\hline \multirow[t]{3}{*}{11} & $\begin{array}{l}\text { Echinorhinus cookei } \\
\text { Pietschmann, } 1928\end{array}$ & $\mathrm{D}$ & Fernández, 1975 & $\mathrm{P}$ \\
\hline & \multicolumn{4}{|l|}{ Order Squaliformes } \\
\hline & Family Dalatidae & & & \\
\hline \multirow[t]{2}{*}{12} & $\begin{array}{l}\text { Isistius brasiliensis } \\
\text { (Quoy \& Gaimard, 1824) }\end{array}$ & $\mathrm{D}$ & García, 2017 & $\mathrm{C}$ \\
\hline & \multicolumn{4}{|l|}{ Family Oxynotidae } \\
\hline \multirow[t]{4}{*}{13} & $\begin{array}{l}\text { Oxynotus caribbaeus } \\
\text { Cervigón, } 1961\end{array}$ & $\mathrm{D}$ & Álvarez-León et al., 2013 & $\mathrm{C}$ \\
\hline & \multicolumn{4}{|l|}{ Superorder Batoidei } \\
\hline & \multicolumn{4}{|l|}{ Order Rajiformes } \\
\hline & \multicolumn{4}{|l|}{ Family Arhynchobatidae } \\
\hline \multirow[t]{2}{*}{1} & $\begin{array}{l}\text { Bathyraja spinosissima } \\
\text { (Beebe \& Tee-Van, 1941) }\end{array}$ & $\mathrm{D}$ & Rubio, 1987 & $\mathrm{P}$ \\
\hline & \multicolumn{4}{|l|}{ Family Rajidae } \\
\hline 2 & $\begin{array}{l}\text { Dactylobatus armatus } \\
\text { Bean \& Weed, } 1909\end{array}$ & $\mathrm{C}$ & McEachran \& Carvalho, 2002 & $\mathrm{C}$ \\
\hline \multirow[t]{3}{*}{3} & $\begin{array}{l}\text { Rajella purpuriventralis } \\
\text { (Bigelow \& Schroeder, 1962) }\end{array}$ & $\mathrm{D}$ & Mejía-Falla et al., 2007 & C \\
\hline & \multicolumn{4}{|l|}{ Order Myliobatiformes } \\
\hline & \multicolumn{4}{|l|}{ Family Gymnuridae } \\
\hline 4 & $\begin{array}{l}\text { Gymnura altavela } \\
\text { (Linnaeus, } 1758 \text { ) }\end{array}$ & $\mathrm{D}$ & Álvarez \& Barreto, 1975 & C \\
\hline
\end{tabular}




\begin{tabular}{|c|c|c|c|c|}
\hline 5 & $\begin{array}{l}\text { Gymnura micrura } \\
\text { (Bloch \& Schneider, 1801) }\end{array}$ & D & Mejía-Falla et al., 2007 & $\mathrm{C}$ \\
\hline & \multicolumn{4}{|l|}{ Family Mobulidae } \\
\hline 6 & $\begin{array}{l}\text { Mobula bypostoma } \\
\text { (Bancroft, 1831) }\end{array}$ & $\mathrm{D}$ & Dahl, 1958 & $\mathrm{C}$ \\
\hline 7 & $\begin{array}{l}\text { Mobula mobular } \\
\text { (Bonnaterre, 1788) }\end{array}$ & $\mathrm{C}$ & $\begin{array}{l}\text { McEachran \& Notarbatolo-di } \\
\text { Sciara, } 1995\end{array}$ & $\mathrm{P}$ \\
\hline \multirow[t]{2}{*}{8} & $\begin{array}{l}\text { Mobula tarapacana } \\
\text { (Philippi, 1892) }\end{array}$ & $\mathrm{C}$ & $\begin{array}{l}\text { McEachran \& Notarbatolo-di } \\
\text { Sciara, } 1995\end{array}$ & $\mathrm{P}$ \\
\hline & \multicolumn{4}{|l|}{ Family Myliobatidae } \\
\hline 9 & $\begin{array}{l}\text { Myliobatis goodei } \\
\text { Garman, } 1885\end{array}$ & D & Mantilla, 1998 & $\mathrm{P}$ \\
\hline 10 & $\begin{array}{l}\text { Myliobatis longirostris } \\
\text { (Applegate \& Fitch, 1964) }\end{array}$ & $\mathrm{D}$ & Chirichigno, 2001 & $\mathrm{P}$ \\
\hline \multirow[t]{2}{*}{11} & $\begin{array}{l}\text { Aetomylaeus asperrimus } \\
\text { (Gilbert, 1898) }\end{array}$ & D & Mantilla, 1998 & $\mathrm{P}$ \\
\hline & \multicolumn{4}{|l|}{ Family Urotrygonidae } \\
\hline 12 & $\begin{array}{l}\text { Urotrygon reticulata } \\
\text { Miyake \& McEachran, } 1988\end{array}$ & $\mathrm{C}$ & $\begin{array}{l}\text { McEachran \& Notarbatolo-di } \\
\text { Sciara, } 1995\end{array}$ & $\mathrm{P}$ \\
\hline
\end{tabular}

Table 3. List of elasmobranch species cited in scientific documents but without physical or visual support, and considered unlikely for the Colombian Pacific $(\mathrm{P})$ or Caribbean $(\mathrm{C})$, given its known distribution. The type $(C, D)$ and the reference of support are indicated. Species that presented modification in their systematics or taxonomy are shaded in light green.

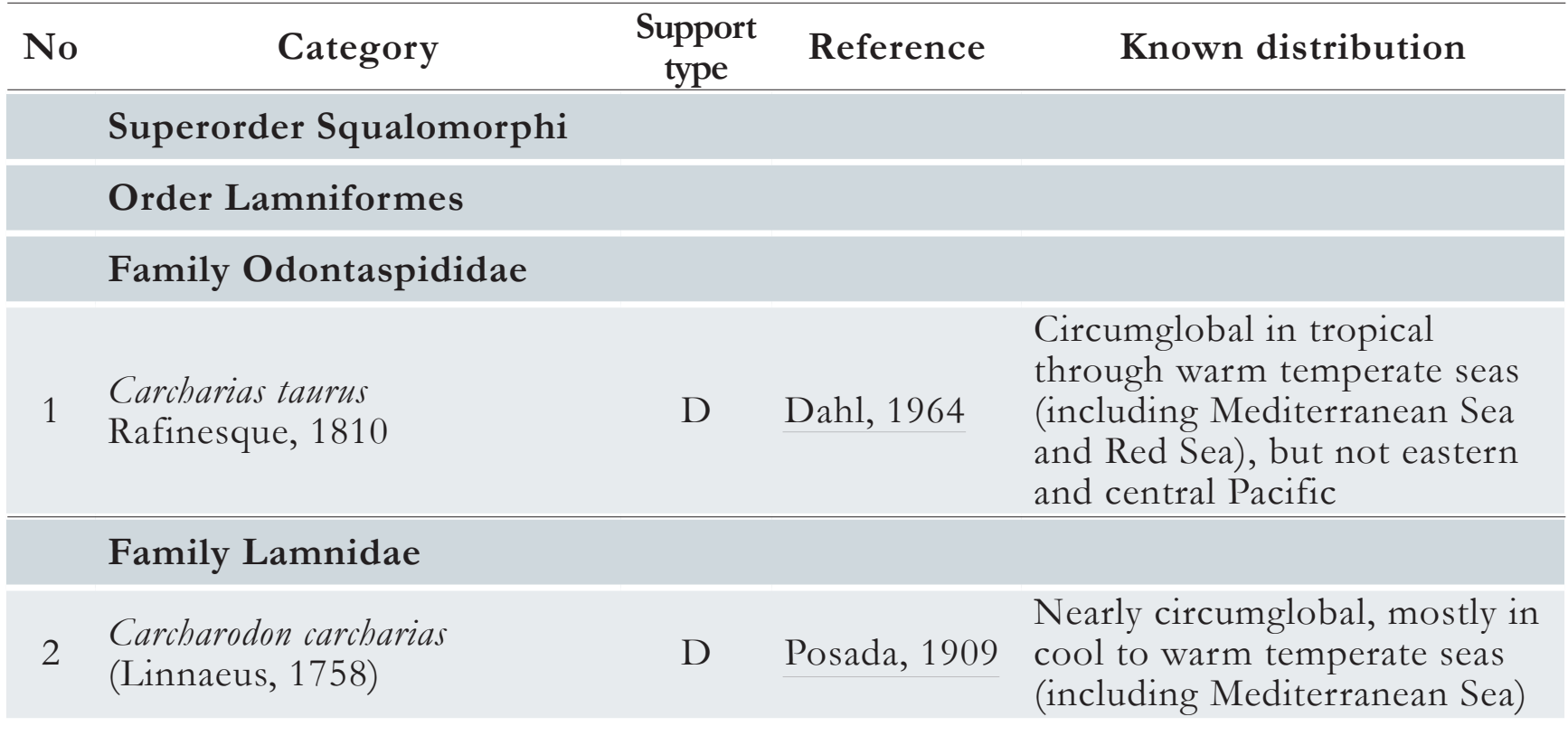




\section{Order \\ Carcharhiniformes}

\section{Family Carcharhinidae}

3 Isogomphodon oxyrbynchus

(Müller \& Henle, 1839)
D Álvarez-León et al., 2013

Central Western Atlantic:

southern Caribbean Sea to Brazil.

\section{Family Scyliorhinidae}

\begin{tabular}{|c|c|c|c|c|}
\hline 4 & $\begin{array}{l}\text { Apristurus brunneus } \\
\text { (Gilbert, 1892) }\end{array}$ & $\mathrm{D}$ & Rubio, 1987 & $\begin{array}{l}\text { British Columbia to Mexico, } \\
\text { possibly to Peru }\end{array}$ \\
\hline 5 & $\begin{array}{l}\text { Cephaloscyllium ventriosum } \\
\text { (Garman, 1880) }\end{array}$ & $\mathrm{D}$ & Álvarez-León et al., 2013 & $\begin{array}{l}\text { Eastern Pacific: Monterey } \\
\text { Bay, California to southern } \\
\text { Mexico; also Chile }\end{array}$ \\
\hline & $\begin{array}{l}\text { Galeus antillensis } \\
\text { Springer, } 1979\end{array}$ & $\mathrm{D}$ & Álvarez-León et al., 2013 & Western Atlantic \\
\hline
\end{tabular}

\section{Family Triakidae}

$7 \begin{aligned} & \text { Galeorbinus galeus } \\ & \text { (Linnaeus, 1758) }\end{aligned} \quad$ D Álvarez-León et al., 2013
8 Mustelus californicus
Gill, 1864

D Álvarez-León et al., 2013

9 Triakis maculata

D Álvarez-León et al., 2013

Temperate waters of Southern Hemisphere;

North Atlantic (including Mediterranean Sea, western Baltic Sea, North Sea); North Pacific

Northern California (U.S.A.) to Gulf of California and Mexico

D Álvarez-León et al., 2013 Southeastern Pacific

\section{Order Squaliformes}

\section{Family Centrophoridae}

Centrophorus tessellatus
Garman, $1906 \quad$ C Compagno, 2002

Indo-West Pacific:

Maldives, southern Japan, Hawaiian Islands, possibly off North America.

11 Centrophorus uyato (Rafinesque, 1810)

D Álvarez-León et al., 2013

\section{Family Etmopteridae}
Etmopterus granulosus
(Günther, 1880)
Etmopterus pusillus
(Lowe, 1839)
D Rubio, 1987
D García, 2017

Southeastern Pacific, southwestern Atlantic, New Zealand area; Indian Ocean doubtful

Cosmopolitan, mostly in temperate seas (including Hawaiian Islands Chain) 


\section{Family Squalidae}

14 Squalus blainville

(Risso, 1827)

15 Squalus mitsukurii

Jordan \& Snyder, 1903

\section{Superorder Batoidei}

\section{Order Torpediniformes}

\section{Family Narcinidae}

1 Discopyge tschudii

Heckel, 1846

D Álvarez-León et al., 2013

Southeastern Pacific, Southwestern Atlantic

\section{Order}

\section{Rhinopristiformes}

\section{Family Platyrhinidae}

2 Platyrhinoidis triseriata (Jordan \& Gilbert, 1880)

D Álvarez-León et al., 2013

Mediterranean Sea, Black Sea, North Atlantic; doubtful from other areas (e.g. Madagascar, Mascarenes, New Caledonia, Japan)

Japan and in tropical through temperate seas

\section{Family}

\section{Trygonorrhinidae}

3 Zapteryx brevirostris

(Müller \& Henle, 1841)

4 Zapteryx exasperata (Jordan \& Gilbert, 1880)
D Álvarez-León et al., 2013

D Rubio \& Ruíz, 1993
Eastern Pacific: California to Mexico

\section{Order Rajiformes}

\section{Family}

\section{Arhynchobatidae}

\section{Batbyraja aguja}

5 (Kendall \& Radcliffe, 1912)

\section{Bathyraja richardsoni} (Garrick, 1961)

7 Bathyraja spinosissima (Beebe \& Tee-Van, 1941)

Pseudoraja fischeri

8 Bigelow \& Schroeder, 1954

9 Sympterygia brevicaudata (Cope, 1877)
D Álvarez-León et al., 2013 Southeastern Pacific: Peru

D Álvarez-León et al., 2013 North Atlantic and southeastern Pacific.

D Álvarez-León et al., 2013

North Pacific: Sea of Okhotsk, Oregon (U.S.A.)

D Álvarez-León et al., 2013 Western Atlantic

D Rubio, 1987
Southeastern Pacific:

Ecuador to Chile (if valid) 


\begin{tabular}{|c|c|c|c|c|}
\hline \multicolumn{5}{|c|}{ Family Rajidae } \\
\hline 10 & $\begin{array}{l}\text { Amblyraja byperborea } \\
\text { (Collett, 1879) }\end{array}$ & C & $\begin{array}{l}\text { McEachran \& Dunn, } \\
1998\end{array}$ & $\begin{array}{l}\text { Cosmopolitan, circumpolar, } \\
\text { North Atlantic, western } \\
\text { Arctic Ocean, and South } \\
\text { Pacific New Zealand and } \\
\text { Tasmania }\end{array}$ \\
\hline 11 & $\begin{array}{l}\text { Beringraja cortezensis } \\
\text { McEachran \& Miyake, } \\
1988\end{array}$ & C & Robertson \& Allen, 2015 & Gulf of California. \\
\hline 12 & $\begin{array}{l}\text { Breviraja colesi } \\
\text { Bigelow \& Schroeder, } \\
1948\end{array}$ & $\mathrm{D}$ & Álvarez-León et al., 2013 & $\begin{array}{l}\text { Western Atlantic: Florida } \\
\text { (U.S.A.), Bahamas and Cuba }\end{array}$ \\
\hline 13 & $\begin{array}{l}\text { Leucoraja garmani } \\
\text { (Whitley, 1939) }\end{array}$ & $\mathrm{D}$ & Álvarez-León et al., 2013 & $\begin{array}{l}\text { Western Atlantic: } \\
\text { Massachusetts to Florida } \\
\text { (U.S.A.) }\end{array}$ \\
\hline \multirow[t]{2}{*}{14} & $\begin{array}{l}\text { Leucoraja lentiginosa } \\
\text { (Bigelow \& Schroeder, 1962) }\end{array}$ & $\mathrm{D}$ & Álvarez-León et al., 2013 & Gulf of Mexico \\
\hline & \multicolumn{4}{|l|}{ Family Gurgesiellidae } \\
\hline \multirow[t]{3}{*}{15} & $\begin{array}{l}\text { Fenestraja ishiyamai } \\
\text { (Bigelow \& Schroeder, 1962) }\end{array}$ & $\mathrm{D}$ & Álvarez-León et al., 2013 & Western Atlantic \\
\hline & \multicolumn{4}{|l|}{ Order Myliobatiformes } \\
\hline & \multicolumn{4}{|l|}{ Family Dasyatidae } \\
\hline 16 & $\begin{array}{l}\text { Fontitrygon geijskesi } \\
\text { (Boeseman, 1948) }\end{array}$ & $\mathrm{D}$ & Álvarez-León et al., 2013 & $\begin{array}{l}\text { Western Central Atlantic: } \\
\text { Suriname to northern Brazil }\end{array}$ \\
\hline 17 & $\begin{array}{l}\text { Hypanus sabinus } \\
\text { (Lesueur, 1824) }\end{array}$ & $\mathrm{D}$ & Dahl, 1958 & $\begin{array}{l}\text { Western Atlantic, from } \\
\text { North Carolina (U.S.A.) } \\
\text { and northern and western } \\
\text { Gulf of Mexico. ICN- } \\
\text { MHN: } 4025 \text { is located in } \\
\text { the Putumayo River and } \\
\text { therefore it was considered } \\
\text { an invalid record }\end{array}$ \\
\hline 18 & $\begin{array}{l}\text { Hypanus say } \\
\text { (Lesueur, 1817) }\end{array}$ & $\mathrm{D}$ & Dahl, 1958 & $\begin{array}{l}\text { Western Atlantic: } \\
\text { Massachusetts (U.S.A.) to } \\
\text { Brazil }\end{array}$ \\
\hline & \multicolumn{4}{|l|}{ Family Myliobatidae } \\
\hline 19 & $\begin{array}{l}\text { Myliobatis freminvillei } \\
\text { Lesueur, } 1824\end{array}$ & $\mathrm{D}$ & Álvarez \& Barreto, 1975 & $\begin{array}{l}\text { Western Atlantic: } \\
\text { Massachusetts (U.S.A.) and } \\
\text { Brazil to Argentina }\end{array}$ \\
\hline & \multicolumn{4}{|l|}{ Family Urotrygonidae } \\
\hline 20 & $\begin{array}{l}\text { Urotrygon microphthalmum } \\
\text { Delsman, } 1941\end{array}$ & $\mathrm{D}$ & Mejía-Falla et al., 2007 & $\begin{array}{l}\text { Western Atlantic: Venezuela } \\
\text { to northeastern Brazil }\end{array}$ \\
\hline 21 & $\begin{array}{l}\text { Urotrygon nana } \\
\text { Miyake \& McEachran, } 1988\end{array}$ & $\mathrm{D}$ & Tobón, 2001 & $\begin{array}{l}\text { Eastern Pacific: Mexico to } \\
\text { Panama }\end{array}$ \\
\hline
\end{tabular}


Table 4. Species that although it has museum number, visual o catch record in Colombian waters, they are considered a bad identification.

\begin{tabular}{|c|c|c|c|}
\hline No & Category & Support & Comments \\
\hline & Superorder Squalomorphi & & \\
\hline & Order Heterodontiformes & & \\
\hline & Family Heterodontidae & & \\
\hline \multirow[t]{3}{*}{1} & $\begin{array}{l}\text { Heterodontus quoyi } \\
\text { (Fréminville, 1840) }\end{array}$ & $\begin{array}{l}\text { PNNG 680, } \\
1250\end{array}$ & $\begin{array}{l}\text { These records were verified by the } \\
\text { authors and belongs to } H \text {. mexicanus. }\end{array}$ \\
\hline & Order Carcharhiniformes & & \\
\hline & Family Carcharhinidae & & \\
\hline 2 & $\begin{array}{l}\text { Carcharbinus signatus } \\
\text { (Poey, 1868) }\end{array}$ & Record SGC & \\
\hline \multirow[t]{4}{*}{3} & $\begin{array}{l}\text { Rhizoprionodon terraenovae } \\
\text { (Richardson, 1837) }\end{array}$ & FLMNH 222229 & $\begin{array}{l}\text { Western Atlantic: Canada to Yucatan, } \\
\text { Mexico. }\end{array}$ \\
\hline & Superorder Batoidei & & \\
\hline & Order Rhinopristiformes & & \\
\hline & Family Rhinobatidae & & \\
\hline \multirow[t]{3}{*}{1} & $\begin{array}{l}\text { Pseudobatos lentiginosus } \\
\text { (Garman, 1880) }\end{array}$ & INV-PEC 8199 & $\begin{array}{l}\text { Western Atlantic: Southeast USA and } \\
\text { coastal regions of the Gulf of Mexico } \\
\text { from North Carolina to Yucatán. }\end{array}$ \\
\hline & Order Rajiformes & & \\
\hline & Family Rajidae & & \\
\hline \multirow[t]{3}{*}{2} & $\begin{array}{l}\text { Dipturus oregoni } \\
\text { (Bigelow \& Schroeder, 1958) }\end{array}$ & García, 2017 & $\begin{array}{l}\text { Western Central Atlantic: apparently } \\
\text { restricted to the Gulf of Mexico. } \\
\text { Capture record with coordinates in Colombia } \\
\text { (R/V Oregon II, 1970) could correspond to } \\
\text { a bad identification. }\end{array}$ \\
\hline & \multicolumn{3}{|l|}{ Order Myliobatiformes } \\
\hline & \multicolumn{3}{|l|}{ Family Dasyatidae } \\
\hline 3 & $\begin{array}{l}\text { Bathytoshia centroura } \\
\text { (Mitchill, 1815) }\end{array}$ & $\begin{array}{l}\text { Catch Anguila et } \\
\text { al. (2016b) }\end{array}$ & $\begin{array}{l}\text { Western Atlantic: Georges Bank and } \\
\text { Cape Cod south to Florida and in the } \\
\text { northeastern Gulf of Mexico (USA), } \\
\text { and in the Bahamas. South of Brazil, } \\
\text { Uruguay and Argentina. }\end{array}$ \\
\hline \multicolumn{4}{|c|}{ Family Urotrygonidae } \\
\hline 4 & $\begin{array}{l}\text { Urotrygon simulatrix } \\
\text { Miyake \& McEachran, } 1988\end{array}$ & $\begin{array}{l}\text { USNM } 222562 \\
222636,222642, \\
222640\end{array}$ & $\begin{array}{l}\text { Eastern central Pacific: known only } \\
\text { from the Gulf of Panama, Panama. }\end{array}$ \\
\hline
\end{tabular}


This review allowed to update the taxonomy and systematics of the group. Modifications to the scientific name of two sharks and 20 rays and skates were identified, as well as changes to some batoid orders, families and genera with exclusive distribution in the American continent (Last et al., 2016 b,c). The most significant results for sharks were: 1) the division of Ginglymostoma cirratum into separate species, with $G$. cirratum for the Atlantic and Caribbean, whereas Pacific populations received the name Ginglymostoma unami. 2) The separation of Carcharbinus porosus as a species exclusively for the Caribbean and the resurrection of $C$. cerdale as the valid species for the Pacific. 3) The description of a new angel shark species (Squatina david) based on specimens from the Colombian Caribbean (holotype), and consequently, according to Vaz \& Carvalho (2018), this species replaces S. dumeril; so that the specimens previously identified as $S$. dumeril possibly correspond to S. david. 4) The first record of the sleeper shark (Somniosus sp.) for deep waters of the southern Caribbean of Colombia; although it was not possible to confirm the species, the authors (Acero-P et al., 2018) remarked that it is highly possible that the specimen could be the Greenland shark, S. microcephalus (Bloch \& Schneider, 1801).

The batoid group presented the greatest quantity of modifications over the past few years, such as: Changes to the genus of the species Torpedo nobiliana and Torpedo tremens that are now valid as Tetronarce nobiliana and Tetronarce tremens (Carvalho et al., 2016a). The union of the old orders Rhinobatiformes and Pristiformes into Rhinopristiformes, as well as the modification of the genus Rhinobatos into the genus Psendobatos for guitarfish from the American continent (Last et al., 2016b). Within this same order, species from the genus Zapteryx were taken out of the Rhinobatidae Family and assigned to the Trygonorrbinidae Family (Last \& Séret, 2016).

Within the Rajidae Family, the genus Raja was modified for neotropical species, and is now recognized as Rostroraja (Last et al., 2016c). The species name Anacanthobatis americanus was changed to Schroederobatis americana (Last et al., 2016c; Séret et al., 2016). Within the Dasyatidae Family, the genus name Dasyatis was changed to Hypanus, valid only for the rays of this family on the American continent (Last et al., 2016d).

The species Manta birostris was moved into the genus Mobula (White et al., 2018), whereas the Aetobatus narinari family name was changed; the family name Aetobatidae was revived, and the species was separated into different species for the Pacific (Aetobatus laticeps) and Atlantic (Aetobatus narinari) (Last et al., 2016). Another significant change occurred for the species Himantura pacifica and $H$. schmardae, which were moved from the family Dasyatidae into the family Potamotrygonidae, 
and were also assigned a new genus and are now recognized as Styracura pacifica and Styracura schmardae (Carvalho et al., 2016b). Finally, Urotrygon asterias, $U$. caudispinosus and $U$. serrula are now synonymous with $U$. munda (Weigmann, 2016).

\section{Conclusions}

The 138 elasmobranch species confirmed for Colombia represented $12.1 \%$ of the known worldwide elasmobranch richness (Weigmann, 2016); this means that Colombia is the third country in terms of elasmobranch diversity in Latin America, behind Mexico (214, del Moral-Flores et al., 2015) and Brazil (165, Rosa \& Gadig, 2014), and before Argentina (105, PAN-Tiburones, 2009) and Chile (92, Lamilla \& Bustamante, 2005). There were also 25 potential species records that were not confirmed for Colombia, which could increase richness to 163 species, representing $15.10 \%$ of worldwide elasmobranch species richness.

Compared with neighboring and/or bordering countries, Colombia has more shark and ray species than Venezuela (74, Cervigón, 1999; Robertson et al., 2015), Ecuador (94, Martínez-Ortiz \& García-Domingo, 2013), and Peru (115, Cornejo et al., 2015). However, it should be noted that the published checklists of Venezuela and Ecuador have not been updated.

Comparing basins, the 90 Caribbean elasmobranch species represented $86.5 \%$ of the species reported for the Greater Caribbean coastal zone (Robertson et al., 2015) and $42.2 \%$ of the species reported for the North West Atlantic (Weigmann, 2016), whereas representativeness was lower for the Pacific basin, with $58.2 \%$ of the species reported by Robertson \& Allen (2015) for the Tropical Eastern Pacific and $43.8 \%$ of the species to the North Eastern Pacific (Weigmann, 2016). Shark and batoid richness for the Colombian Caribbean was greater than that of Venezuela and Panama (Robertson et al., 2015), whereas shark and batoid richness for the Pacific was lower than that reported by Martínez-Ortiz \& García-Domingo (2013) for Ecuador (61 shark species and 43 batoid species), by Cornejo et al. (2015) for Peru (66 shark species and 43 batoids species) and by Espinoza et al. (2018) for Costa Rica (81 elasmobranch species). However, it should be noted that Peru and Ecuador are strongly influenced by fauna from temperate waters of the southern Pacific, which increases significantly their diversity, as they provide species from different biogeographic provinces. Elasmobranch richness for the tropical zone of the Pacific basin of Colombia was greater than that of Panama in both coasts and greater than those of Costa Rica in the Caribbean Sea (Robertson \& Allen, 2015; Espinoza et al., 2018). 
Despite the great species richness found in a regional context, Colombia was far below shark biodiversity hotspots, which have been identified in southeastern Japan, Taiwan, and Australia, with nearly 85 species per $1^{\circ} \times 1^{\circ}$ cell. The elasmobranch endemism level was also very low in Colombian waters in comparison with endemism hotspots identified by Lucifora et al. (2011), which were also located in southern Japan, Taiwan, Australia, as well as southern Brazil and the southeastern United States.

Shark richness values for the Caribbean and Pacific of Colombia agreed to bimodal distribution patterns described by Lucifora et al. (2011), who suggested a greater number of species towards intermediate latitudes of the northern and southern hemispheres than towards the tropics. However, these authors reported species numbers below those found in this study for Colombian Caribbean and Pacific waters. This could be due to the low number of studies on richness carried out in Colombia that are available in scientific journals with large circulation, as most publications have been published in Spanish, resulting in information available at the national level not being visible to researchers from other countries.

This demonstrates that although Colombia cannot be considered an elasmobranch diversity or endemism hotspot globally, there is great biodiversity representation of these species regionally, especially of amphi-American species. This is particularly important as several areas with moderate shark richness have shown high functional richness, suggesting that these species play unique roles, that they are not very redundant and are therefore very important for maintaining the structure and function of marine ecosystems. In this regard, the Pacific and Caribbean coasts of Colombia are one of the 15 hotspots of irreplaceability of endemic threatened marine chondrichthyans identified by Dulvy et al. (2014). These authors identified these hotspots to guide conservation priorities given that they contain the most unique chondrichthyan biodiversity.

Therefore, detailed knowledge of the richness and distribution of sharks and rays is an important tool for planning diversity management and conservation measures, especially in this type of group that includes an important number of highly migratory species. Finally, if the 11 species of freshwater stingrays confirmed for Colombia (Lasso et al., 2016; Do Nascimiento et al., 2017) are added to marine elasmobranch species, total richness would be 174 species, representing $15.3 \%$ of worldwide richness, a value that confirms the importance of Colombia for the diversity of this taxonomic group. 


\section{Conflict of interest}

The authors declare having no conflict of interest

\section{References}

Acero-PA, Franke R. Nuevos registros de peces cartilaginosos del Parque Nacional Natural Gorgona (Pacífico Colombiano), II. Rayas y descripción de una nueva especie. In: La Isla de Gorgona, Nuevos estudios Biológicos. Biblioteca José Jerónimo Triana / Instituto de Ciencias Naturales, Museo de Historia Natural, Universidad Nacional de Bogotá. 11: 9-21, 1995.

Acero-PA, Cánter-Ríos D, Polanco-F A. Identidad problemática del tiburón martillo ojichico (Carcharhiniformes: Sphyrna) existente en Colombia. Boletin de Investigaciones Marinas y Costeras, 36(1): 321 326, 2007.

Acero-PA, Tavera JJ, Anguila R, Hernández-Beracasa L. A New Southern Caribbean Species of Angel Shark (Chondrichthyes, Squaliformes, Squatinidae), including phylogeny and tempo of diversification of American species, Copeia, 104(2): 577-585, 2016.

doi: 10.1643/CI-15-292

Acero-PA, Polo-Silva CJ, León J, Puentes V. First report of a sleeper shark (Somniosus sp.) in the southern Colombian Caribbean, Journal of Applied Ichthyology, 1-3, 2018.

doi: 10.1111/jai.13712

Álvarez R, Barreto J. Catálogo de peces. Rayas, Informe Museo del Mar, 14: 1-40, 1975.

Álvarez-León R, Orozco-Rey RH, Páramo-Fonseca ME, RestrepoSantamaría D. Lista de los peces fósiles y actuales de Colombia: Nombres científicos válidos, distribución geográfica, diagnosis de referencia y nombres comunes e indígenas. 1ra edición. Eco prints diseño gráfico y Audiovisual LTDA, Colombia 2013.

Anguila R, Nieto-Alvarado LE, Hernández-Beracasa L. Nuevos registros de peces de esqueleto cartilaginoso para el Caribe colombiano y uno como ampliación de su distribución geográfica en el Caribe colombiano para Bocas de Ceniza, departamento de Atlántico, Colombia, Boletín de Investigaciones Marinas y Costeras, 45(2): 361-373, 2016 a.

Anguila R, Nieto-Alvarado LE, Narváez-Barandica JC, HernándezBeracasa L, Acero-P A. Ampliación geográfica del tiburón siete branquias o bocadulce Heptranchiasperlo Bonnaterre (Hexanchiformes: Hexanchidae) para el caribe continental colombiano, Boletín de Investigaciones Marinas y Costeras, 45(2): 355-360, $2016 \mathrm{~b}$. 
Ballesteros C, Castro E. La pesquería industrial de tiburones en el Archipiélago de San Andrés, Providencia y Santa Catalina: Una primera aproximación. Secretaria de agricultura y pesca. 2006

Caldas J, Santos A, Acero-P A. Tiburones viuda (Mustelus, Triakidae) del Archipiélago de San Andrés, Providencia y Santa Catalina, Caribe colombiano. En: Universidad Nacional de Colombia. Ed. Investigación y desarrollo de territorios promisorios. Contribución Ciencias del Mar Colombia, Bogotá, 2004.

Carvalho MR de, Last PR, Séret B. Torpedo Rays. Family Torpedinidae. In: Last PR, White WT, Carvalho MR de, Séret B, Stehmann MFW, Naylor GJP. (Eds). Rays of the World, CSIRO Publishing, Comstock Publishing Associates, Australia 2016 a.

Carvalho MR de, Loboda TS, da Silva JPCB. A new subfamily, Styracurinae, and new genus, Styracura, for Himantura schmardae (Werner, 1904) and Himantura pacifica (Beebe \& Tee-Van, 1941) (Chondrichthyes: Myliobatiformes), Zootaxa, 4175 (3): 201-221, 2016b.

doi: $10.11646 /$ zootaxa.4175.3.1

Castro JI. Resurrection of the name Carcharbinus cerdale, a species different from Carcharhinus porosus, AQUA. International Journal of ichthyology, 17(1): 1-10, 2011.

Cervigón F, Alcalá A. Los peces marinos de Venezuela. Parte V: Elasmobranquios. Fundación Científica Los Roques, Venezuela 1999.

Chirichigno N, Cornejo M. Catálogo comentado de los peces marinos del mar del Perú. Instituto del Mar del Perú, Perú 2001.

Compagno LJV, Krupp F, Schneider W. Tiburones. En: W Fischer, F Krupp, W Schneider, C Sommer, K Carpenter y V Niem (Eds). Guía para la identificación de especies para los fines de la pesca, Pacífico Centro Oriental. FAO, Roma 1995.

Compagno LJV. Sharks. En: Carpenter, K. y P. Oliver (Eds.). Species identification sheets for fishery purposes, Western Central Atlantic. FAO, Roma 2002.

Cornejo R, Vélez-Zuazo X, González-Pestaña A, Kouri CJ, Mucientes G. An updated checklist of Chondrichthyes from the southeast Pacific off Peru, Check List, 11(6): 1809, 2015.

doi: 10.15560/11.6.1809 
Dahl G. Los peces del río Sinú. Secretaría de Agricultura y Ganadería, Córdoba, Colombia 1958.

Dahl G. Los peces cartilaginosos de la Bahía de Cispatá y del estuario del río Sinú, Revista Academia Colombiana Ciencias Exactas Físicas y Naturales, 46: 175-195, 1964.

Del Moral-Flores LF, Morrone JJ, Alcocer-Durand J, EspinosaPérez H, Pérez-Ponce De León G. Lista patrón de los tiburones, rayas y quimeras (Chondrichthyes, Elasmobranchii, Holocephali) de México, Arxius de Miscel-lània Zoològica, 13: 47-163, 2015.

DoNascimiento C, Herrera-Collazos EE, Herrera-R GA, OrtegaLara A, Villa-Navarro FA, Usma-Oviedo JS, Maldonado-Ocampo JA. Checklist of the freshwater fishes of Colombia: a Darwin Core alternative to the updating problem, ZooKeys, 708: 25-138, 2017.

doi: $10.3897 /$ zookeys.708.13897

Dulvy NK, Fowler SL, Musick JA, Cavanagh R, Kyne P, Harrison LR, Carlson JK, Davidson LNK, Fordham SV, Francis MP, Pollock CM, Simpfendorfer CA, Burgess GH, Carpenter KE, Compagno LJV, Ebert DA, Gibson C, Heupel MR, Livingstone SR, Sanciangco JC, Stevens JD, Valenti S, White WT.. Extinction risk and conservation of the world's sharks and rays, eLife, 3:e00590. 2014.

doi: 10.7554/eLife.00590

Espinoza M, Díaz E, Angulo A, Hernández S, Clarke TM. Chondrichthyan Diversity, Conservation Status, and Management Challenges in Costa Rica, Frontiers in Marine Science, 5:85. 2018.

doi: $10.3389 /$ fmars.2018.00085

Fernández P. Estudio biológico y pesquero de algunas especies de tiburones del Pacífico colombiano pertenecientes al Orden Lamniformes. Tesis Biol. Mar., Univ. Jorge Tadeo Lozano, Bogotá, 1975.

Gámez-Barrera D, Nieto-Alvarado LE, Morón-Granados E, Caldas JP, Correa JL. Primer registro del tiburón mako aleta larga, Isurus paucus Guitart (Chondrichthyes: Lamnidae), para el Caribe colombiano, Boletín de Investigaciones Marinas y Costeras, 41(2): 485490, 2012.

García CB. What do we know about soft-bottom elasmobranch species richness in the Colombian Caribbean and of its spatial distribution? Regional Studies in Marine Science, 9: 62-68, 2017.

doi: 10.1016/j.rsma.2016.11.006 
Gómez-Rodríguez S, Caldas JP, Acero-P A, Martínez-Silva MA, Saenz-Okuyama P, Lasso CA, Lasso-Alcalá OM. Geographic distribution and conservation status of sawfish Pristis spp (Pristiformes: Pristidae) in the southern Caribbean Sea, Biota Colombiana,15(Supl. 1): 109-117, 2014.

Grijalba-Bendeck LM. Acevedo K. Mitsukurina owstoni Jordan (Chondrichthyes: Mitsukurinidae) primer registro para el Caribe colombiano, Boletín de Investigaciones Marinas y Costeras, 38(1): 211215, 2009.

Lamilla J, Bustamante C. Guía para el reconocimiento de: tiburones, rayas y quimeras de Chile, Oceana, Chile, 2005.

Lasso CA, Rosa RS, Morales-Betancourt MA, Garrone-Neto D, Carvalho M. (Eds.). XV. Rayas de agua dulce (Potamotrygonidae) de Suramérica. Parte II. Colombia, Brasil, Perú, Bolivia, Paraguay, Uruguay y Argentina, Serie Editorial Recursos Hidrobiológicos y Pesqueros Continentales de Colombia, Instituto de Investigación de Recursos Biológicos Alexander von Humboldt, Colombia 2016.

Last PR, Séret B. Banjo Rays. Family Trygonorrhinidae. In: Last PR, White WT, Carvalho MR de, Séret B, Stehmann MFW, Naylor GJP. (Eds). Rays of the World, CSIRO Publishing, Comstock Publishing Associates, Australia 2016.

Last PR, White WT, de Carvalho MR, Séret B, Stehmann MFW, Naylor GJP (Eds.). Rays of the World, CSIRO Publishing, Comstock Publishing Associates, Australia 2016a.

Last PR, Seret B, Naylor GJP. A new species of guitarfish, Rhinobatos borneensis sp. nov. with a redefinition of the family-level classification in the order Rhinopristiformes (Chondrichthyes: Batoidea), Zootaxa, 4117(4): 451-475, 2016 b.

doi: 10.11646/zootaxa.4117.4.1

Last PR, Weigmann S, Yang L. Changes to the nomenclature of the skates (Chondrichthyes: Rajiformes). In: Last PR, Yearsley GK (Eds). Rays of the world. Supplementary information, CSIRO Special Publication, $2016 \mathrm{c}$.

Last PR, Naylor GJP, Manjaji-Matsumoto BM. A revised classification of the family Dasyatidae (Chondrichthyes: Myliobatiformes) based on new morphological and molecular insights, Zootaxa, 4139(3): 345-368, 2016d.

doi: 10.11646/zootaxa.4139.3.2 
Lucifora LO, García VB, Worm B. Global Diversity hotspots and conservation priorities for sharks, PLoS ONE, 6(5): e19356, 2011. doi: 10.1371/journal.pone.0019356

Mantilla L. Lista de Especies Elasmobranquios de Colombia, Revista de Fenología y Anatomia, Vol I. 19-08-2006.

https://www.researchgate.net/publication/230880963_Lista_de_ Especies_Elasmobranquias_de_Colombia

Martínez A. Peces deportivos de Colombia, Editorial Fondo Cultural Cafetero, Colombia 1978.

Martínez-Ortiz J, García-Domínguez, M. Guía de campo Condrictios del Ecuador. Quimeras, tiburones y rayas, Ministerio de Agricultura, Ganadería, Acuiacultura y Pesca (MAGPA)/ Vice-Ministerio de Acuacultura y Pesca (VMAP)/ Subsecretaría de Recursos Pesqueros, Ecuador, 2013.

McEachran J, Carvalho de MR. Batoid Fishes. En: Carpenter K, Oliver P (Eds). Species identification sheets for fishery purposes, Western Central Atlantic, FAO, Roma 2002.

McEachran J,Dunn K. Phylogenetic analysis of skates, a morphologically conservative clade of elasmobranches (Chondrichthyes: Rajidae), Copeia, 1998(2): 271-290, 1998.

doi: $10.2307 / 1447424$

McEachran J, di Sciara N. Peces Batiodeos. En: Fischer W, Krupp F, Schneider W, Sommer C, Carpenter K, Niem V. (Eds). Guía para la identificación de especies para los fines de la pesca, Pacífico Centro Oriental. FAO, Roma, 1995.

Mejía-Falla PA, Navia AF. New records of Urobatis tumbesensis (Chirichigno \& McEachran, 1979) in the Tropical Eastern Pacific, Pan-American Journal of Aquatic Science, 4(3): 255-258, 2009.

Mejía-Falla PA, Navia AF, Mejía LM, Acero A, Rubio EA. Tiburones y rayas de Colombia (Pisces: elasmobranchii): lista actualizada, revisada y comentada, Boletín de Investigaciones Marinas y Costeras, 36: 111-149, 2007.

Mejía-Falla PA, Navia AF, Puentes V. Guía para la identificación de especies de tiburones, rayas y quimeras de Colombia, Ministerio de Ambiente y Desarrollo Sostenible, Corporación para el Desarrollo Sostenible del Archipiélago de San Andrés, Providencia y Santa Catalina - CORALINA, Gobernación de San Andrés, Providencia y Santa Catalina, Fundación SQUALUS, Colombia 2011. 
Mercado J. Lista de peces cartilaginosos en el litoral Atlántico y Pacífico colombiano, Inderena. Recursos Hidrobiológicos, 3: 64-76, 1990.

Navia AF, Mejía-Falla PA, Hleap JS. Zoogeography of elasmobranchs in the Colombian Pacific Ocean and Caribbean Sea, Neotropical Ichthyology, 14(2): e140134, 2016.

doi: 10.1590/1982-0224-20140134

Naylor GJP, Caira JN, Jensen K, Rosana KAM, Straube N, Lakner C, Carrier, J.C. Elasmobranch Phylogeny: A Mitochondrial Estimate Based on 595 Species. In: Musick JA, Heithaus MR (Eds.) Biology of sharks and their relatives (2 ed.), CRC Press, USA 2012.

Nieto L, Arévalo J, Acero-P A. 2003. Primer registro del tiburón zorro Alopias superciliosus Lowe 1839 (Pisces: Alopiidae) para el PNN Tayrona. En: ACICTIOS (Eds), Memorias VII Simposio Colombiano de Ictiología, Montería, 2003.

Ospina F. Cuando los tiburones atacan. Editorial Carrera Séptima. 2001.

Posada A. Estudios científicos. Imprenta oficial, Medellín, III, Colombia 1909.

PAN Tiburones. Plan de acción nacional para la conservación y el manejo de condrictios en la República Argentina. Consejo Federal Pesquero, Subsecretaría de Pesca y Acuicultura (SSPyA), Secretaría de Ambiente y Desarrollo Sustentable y Ministerio de Relaciones Exteriores, Comercio Internacional y Culto. Argentina 2009.

Robertson DR, Allen GR. Peces Costeros del Pacífico Oriental Tropical: Sistema de Información en línea. Versión 2.0 Instituto Smithsonian de Investigaciones Tropicales, República de Panamá 2015.

Robertson DR, Peña EA, Posada JM, Claro R. Peces costeros del Gran Caribe: sistema de información en línea. Versión 1.0 Instituto Smithsonian de Investigaciones Tropicales, República de Panamá 2015.

Rosa RS, Gadig OBF. Conhecimiento da diversidade dos Chondrichthyes marinos no Brasil; a contribuçáo de José Lima de Figueiredo, Arquivos de Zoologia, 45: 89-104. 2014.

Ross R. Scheafer F. Süßwasserrochen Freshwater rays. MörfeldenWalldorf, AQUALOG, Germany 2000. 
Rubio EA. Lista sistemática de peces costeros y de profundidad del Pacífico colombiano, Facultad de Ciencias Universidad del Valle, Colombia 1987.

Rubio E, Ruíz W. Primer registro de Zapteryx exasperata (Batoideos:Rajiformes: Rhinobatidae) en la costa del Pacífico de Colombia, Revista de Ciencias Universidad del Valle, 1: 11-16, 1993.

Rubio EA, Pedraza M, Zapata LA. Primer registro del tiburón perro Centroscyllium nigrum (Chondrichthyes: Squalidae) en aguas del Pacífico colombiano, Gayana, 69(1): 113-117, 2005.

Skomal GB, Zeeman Si, Chisholm JH, Summers EL, Walsh HJ, McMahon KW, Thorrold SR. Transequatorial Migrations by Basking Sharks in the Western Atlantic Ocean, Current Biology, 19(12): 1019-1022, 2009.

doi: 10.1016/j.cub.2009.04.019

Séret B, Last PR, Weigmann S, Stehmann FW. Legskates. Family Anacanthobatidae, pp 494-508. In: Last PR, White WT, Carvalho MR de, Séret B, Stehmann MFW, Naylor GJP. (Eds). Rays of the World, CSIRO Publishing, Comstock Publishing Associates, Australia 2016.

Vaz DFB, Carvalho MR de. New species of Squatina (Squatiniformes: Squatinidae) from Brazil, with comments on the taxonomy of Angel sharks from the Central and Northwestern Atlantic, Copeia, 106(1): 144-160, 2018.

doi: 10.1643/CI-17-606

Weigmann S. Annotated checklist of the living sharks, batoids and chimaeras (Chondrichthyes) of the world, with a focus on biogeographical diversity, Journal of Fish Biology, 88(3): 837-1037. 2016.

doi: $10.1111 /$ jfb.12874

White WT, Naylor GJP. Resurrection of the family Aetobatidae (Myliobatiformes) for the pelagic eagle rays, genus Aetobatus, Zootaxa, 4139(3): 435-438, 2016.

doi: 10.11646/zootaxa.4139.3.10

White WT, Last PR. Eagle Rays, Family Myliobatidae. In: Last PR, White WT, Carvalho MR de, Séret B, Stehmann MFW, Naylor GJP (Eds.). Rays of the World, CSIRO Publishing, Comstock Publishing Associates, Australia 2016a. 
White WT, Last PR. Pelagic Eagle Rays, Family Aetobatidae. In: Last PR, White WT, Carvalho MR de, Séret B, Stehmann MFW, Naylor GJP (Eds.). Rays of the World, CSIRO Publishing, Comstock Publishing Associates, Australia 2016b.

White WT, Last PR. Devilrays, Family Mobulidae. In: Last PR, White WT, Carvalho MR de, Séret B, Stehmann MFW, Naylor GJP (Eds.). Rays of the World, CSIRO Publishing, Comstock Publishing Associates, Australia 2016c.

White WT, Corrigan S, Yang L, Henderson AC, Bazinet AL, Swofford DL, Naylor GJP. Phylogeny of the manta and devilrays (Chondrichthyes: Mobulidae), with an updated taxonomic arrangement for the family, Zoological Journal of the Linnean Society, 182(1): 50-75, 2018.

doi: 10.1093/zoolinnean/zlx018 


\section{Lista de chequeo de elasmobranquios marinos de Colombia}

Resumen. Se llevó a cabo una revisión y actualización de los registros de especies de tiburones y rayas de aguas marinas colombianas. En la literatura se había registrado un total de 206 especies, de las cuales 138 (76 tiburones y 62 rayas) se habian podido confirmar con registros de museo, capturas, fotografías y videos. No se encontró evidencia de 25 especies, pero su distibución incluye países vecinos, por lo que fueron clasificadas como "posible basada en la distribución". Treinta y seis especies se clasficaron como "improbable basada en la distribución" y siete más se consideraron identificaciones erróneas. El inventario de especies confirmadas incluye 57 géneros (30 tiburones y 27 géneros batoideos) y 34 familias (18 tiburones y 16 familias batoideas). De modo notable, hubo una adición de 26 especies confirmadas desde 2007, así como numerosas modificaciones de la sistemática y taxonomía del grupo, especialmente para batoideos. El número total de elasmobranquios confirmados representó el $12.1 \%$ de las especies conocidas en el mundo, y podría aún alcanzar el $14.5 \%$, lo cual indica que Colombia tiene una de las faunas de peces cartilaginosos más ricas de Latinoamérica, después de México y Brasil. Esto demuestra que, aunque Colombia no puede ser considerada un punto caliente de biodiversidad de elasmobranquios o un sitio de endemismo de elasmobranquios a nivel global, sí tiene una buena representatividad de la biodiversidad de estas especies a nivel regional, especialmente respecto a especies anfi-americanas.

Palabras clave: biodiversidad; tiburones y batoideos; lista de especies; riqueza. 


\section{Lista de verificação de elasmobrânquios da Colômbia}

Resumo Realizou-se uma revisão e atualização dos registros de espécies de tubarões e arraias de água marinha colombianas. Na literatura se encontrava registro de um total de 206 espécies, das quais 138 (76 tubarões e 62 arraias) foram possíveis de confirmação por meio de registros de museus, capturas, fotografias e vídeos. Não se encontrou evidencia de 25 espécies, mas suas distribuições incluem países vizinhos, sendo então classificadas como "possível com base na distribuição". Trinta e seis espécies se classificaram como "improváveis com base na distribuição" e outras sete se consideraram identificações errôneas. O inventário de espécies confirmadas inclui 57 gêneros (30 tubarões e 27 gêneros de batóides) e 34 famílias (18 tubarões e 16 famílias batóides). Notavelmente, houve uma adição de 26 espécies confirmadas desde 2007, assim como numerosas modificações da sistemática e taxonomia do grupo, especialmente para batóides. O número total de elasmobrânquios confirmados representou um $12.1 \%$ das espécies conhecidas no mundo, e poderia ainda alcançar $14.5 \%$, o que indica que Colômbia tem uma das faunas mais rica da América Latina em peixes cartilaginosos, depois de México e Brasil. Isto mostra que Colômbia, ainda que possa não ser considerada um ponto de referência de biodiversidade de elasmobrânquios ou um local de endemismo de elasmobrânquios a nível global, possui uma boa representatividade da biodiversidade de estas espécies a nível regional, especialmente com respeito a espécies anfi-americanas.

Palavras-chave: biodiversidade; tubarões e batóides; lista de espécies; riqueza. 


\section{Andrés Felipe Navia}

His main research interest is directed to the evaluation of the ecological function of top predators in marine ecosystems and the effect of fishing on food webs. He has conducted research about determination of the relationship between life history characteristics of elasmobranchs and their vulnerability. He recently initiated studies on the essential habitats of elasmobranchs and ecological processes that determine their richness and distribution.

ORCID: https:/ /orcid.org/0000-0002-6758-7729

\section{Paola Andrea Mejía-Falla}

Her research focused on the life history strategies and demographics of elasmobranchs, as well as to assess the effect of fishing on such population parameters. She has conducted research on reproduction, age and growth of various species of elasmobranchs, both marine and freshwater. She has initiated studies on essential habitats of elasmobranches and on the macro-ecological processes that determine the distribution of these species.

ORCID: https://orcid.org/0000-0003-2220-6969 